\title{
An equality test across nonparametric regressions*
}

\author{
Pascal Lavergne ${ }^{\dagger}$ \\ INRA-ESR, Toulouse
}

March 1997, revised May 2000

\begin{abstract}
We propose a procedure for testing the equality of several nonparametric multivariate regressions. We allow the regressors' designs and the number of observations to differ across subsamples. The division into subsamples is defined through a variable $C$ which can be either fixed or random. For a random $C$, our procedure is a general test of significance for qualitative variables in a nonparametric regression. For a fixed $C$, our procedure provides a "nonparametric analysis of covariance," which is valid for cross-section or panel data. In both cases, the test is a one-sided normal test and is consistent against all alternatives.

Keywords: Hypothesis testing, Nonparametric methods, Qualitative variables, Covariance analysis.

JEL classification: Primary C52; Secondary C14.
\end{abstract}

\footnotetext{
${ }^{*}$ Part of this work was done while visiting Universidad Carlos III de Madrid. Financial support from INRA and from the European Commision through research training grant ERBFMBICT961595 is gratefully acknowledged. A previous version of this paper was presented at Universidad Carlos III and CEMFI (Madrid), University Toulouse 1, DELTA (Paris), Camp Econometrics 98 (Catalina Island) and ESEM 98 (Berlin). I thank the participants, and especially Manuel Arellano, Richard Blundell, Jean-Marc Robin, Alban Thomas and Quang Vuong, as well as the editor and two referees, for helpful comments.

${ }^{\dagger}$ Corresponding address: INRA-ESR, BP 27, 31326 CASTANET-TOLOSAN Cedex FRANCE. E-mail address: lavergne@toulouse.inra.fr
} 


\section{Introduction}

A classic problem in econometrics is determining whether the form of a regression function remains the same for two or more separate subsamples. Beginning with Chow's (1960) work, a lot of attention has been devoted in the econometric literature to testing equality of regression functions. The related procedures have been used for various economic problems, such as testing for gender or race discrimination in earnings functions, testing for stability over time of economic relationships, testing of disequilibrium models or testing for switching firms' strategies in microeconometric models derived from game theory. Classical procedures assume a parametric form, usually a linear one, for the regression functions under test. As is well-known, specifying an incorrect parametric form can lead to serious errors in inference. Indeed, rejection of the equality hypothesis can be solely due to misspecification of the model. Reversely, overacceptance of the null hypothesis can appear as a consequence of misspecification. Therefore, it is advisable to use a testing procedure free of any parametric assumption.

The issue of comparing regression curves in a nonparametric context has been first addresed in the particular setup of two subsamples with a one-dimensional regressor. ${ }^{1}$ When the regressor's designs are identical, it is possible to use the differences in the dependent variable between the two subsamples to build a test statistic. Hall and Hart (1990) propose a Cramer-von-Mises type statistic while Delgado (1993) studies a Kolmogorov-Smirnov type statistic. Alternatively, one can directly use the mean squared difference between nonparametric regression estimates. This idea has been worked out in the fixed design case by King, Hart and Wehrly (1991), who propose a test under the assumption of normal residuals, and by Härdle and Marron (1990), who consider testing whether two curves are equal up to a parametric transformation. Recent work focuses on specific extensions of the initial setup. A first extension deals with more than two subsamples. Young and Bowman's (1995) test compares several nonparametric regressions depending on a one-dimensional random variable with normal residuals. Fan and Lin (1998) propose testing procedures based on the adaptive Neyman and the wavelet thresholding approaches for comparing multiple curves indexed by time. A second extension deals with non-identical designs. Kulasekera (1995) extends Hall and Hart's procedure to this case. Pinkse and Robinson (1995) generalizes some of Härdle and Marron's (1990) results to random designs and give some guidelines for testing whether two regression functions are similar up to a linear transforma-

\footnotetext{
${ }^{1}$ Testing equality of nonparametric density functions is a closely related problem, see e.g. Mammen (1992).
} 
tion. Hall, Huber and Speckman (1997) build covariate-matching based tests that are taylored against the specific alternative that one of the regression function is always greater than the other. Koul and Schick (1997) propose analogous procedures that incorporate smoothing techniques. Matching is actually used in an earlier illuminating paper by Quade (1982), who deals simultaneously with non-identical designs and multiple subsamples. Other authors considering a general setup are Baltagi, Hidalgo and Li (1996) on testing for poolability of panel data, Munk and Dette (1998) and Yatchew (1999), whose test is based on the comparison of residual variance estimators.

In applied econometrics, we often consider more than one explanatory variable and deal simultaneously with more than two subsamples. More crucially, it is scarcely the case that we have control on the design of explanatory variables. Furthermore, the division into subsamples is also frequently governed by a random variable. In view of practical use in econometrics, we propose a general asymptotic joint test of equality across nonparametric regressions that is consistent against any alternative. It extends previous work in many directions. First, we allow for different unknown data distributions across subsamples. Second, we also allow for any dimension of the explanatory variables. Third, we deal with any number of subsamples. Fourth, we consider random explanatory variables and allow the regressors' designs and the number of observations to differ across subsamples. Fifth, in contrast to previous work that exclusively focuses on the case where the division into subsamples is defined through a fixed variable $C$, we consider as a leading case the situation where a random qualitative variable $C$ defines the split into different subsamples, as frequently arises in economic applications. We subsequently extend our procedure to the usual setup.

When $C$ is fixed, our procedure provides a "nonparametric analysis of covariance" that has numerous potential applications in and outside the field of econometrics. When $C$ is random, our procedure is a general significance test for qualitative variables in a nonparametric regression. This supplements previous work on testing for omitted continuous variables in nonparametric regression, see Aït-Sahalia, Bickel and Stoker (1994), Fan and Li (1996), Gozalo (1995), Lavergne and Vuong (2000) and $\mathrm{Li}(1999) .^{2}$ However, our analysis has a distinctive feature with respect to previous work on smooth tests for omitted variables. Indeed, our null and alternative models

\footnotetext{
${ }^{2}$ More generally, our test is in line with smooth specification tests of parametric models previously proposed in the literature, see Delgado, Dominguez and Lavergne (1998), Härdle and Mammen (1993), Zheng (1996) and some of the previous references.
} 
have the same effective dimension due to the discrete nature of $C$, whereas previous work on nonparametric significance testing compare models with different dimensions. ${ }^{3}$ Hence in our setup, nonparametric estimators of the null and the alternative models have similar rates of convergence and both affect the asymptotic distribution of the test statistic, in contrast to what happens when models have different dimensions.

The paper is organised as follows. In Section 2, we consider the leading case where the splitting variable is random. We set up our testing framework and we derive the basic statistic for testing equality of nonparametric regression functions. We characterize its asymptotic distribution not only under the null hypothesis but also under a sequence of local alternatives. We then derive a consistent testing procedure and discuss its implementation. In Section 3, we treat the case of a fixed splitting variable and relate it to a nonparametric analysis of covariance. We show how the assumptions of Section 2 can be weakened to deal with cross-section and panel data. Section 4 studies the small sample behavior of our test through some simulation experiments. The Conclusion summarizes our main findings. All the proofs are relegated to Appendices.

\section{Case of a random $C$}

\subsection{The testing framework}

Let $C$ be a discrete variable on $C=\{1, \ldots, \bar{C}\}$, with corresponding strictly positive probabilities $p_{1}, \ldots, p_{\bar{C}}$. Let $\left\{\left(C_{i}, X_{i}, Y_{i}\right), i=1, \ldots, n\right\}$ be a sample of i.i.d. observations from $(C, X, Y)$ taking values on $\boldsymbol{C} \times \mathbb{R}^{p} \times \mathbb{R}$. Consider the general regression model

$$
Y_{i}=R\left(X_{i}, C_{i}\right)+U_{i}, \quad E\left[U_{i} \mid X_{i}, C_{i}\right]=0 \quad i=1, \ldots, n,
$$

where $R(\cdot, \cdot)$ denotes the regression function of $Y$ on $X$ and $C$. For any $c$, a nonparametric kernel estimator of $f_{c}(\cdot)$, the conditional density of $X$ given $C=c$, is

$$
f_{n, c}(x)=\left(n_{c} h_{c}^{p}\right)^{-1} \sum_{i=1}^{n} K\left(\frac{x-X_{i}}{h_{c}}\right) \mathbb{I}\left[C_{i}=c\right], \quad \forall x \in \mathbb{R}^{p},
$$

\footnotetext{
${ }^{3}$ This is also true for smooth specification tests of parametric regression models.
} 
where $n_{c}=\sum_{i=1}^{n} \mathbb{I}\left[C_{i}=c\right], K(\cdot)$ is a kernel on $\mathbb{R}^{p}$ and $h_{c}$ is a bandwidth parameter converging to zero. ${ }^{4}$ A nonparametric kernel estimator of $R(\cdot, c)$ is obtained as

$$
R_{n}(x, c)=\frac{\left(n_{c} h_{c}^{p}\right)^{-1} \sum_{i=1}^{n} Y_{i} K\left(\frac{x-X_{i}}{h_{c}}\right) \mathbb{I}\left[C_{i}=c\right]}{f_{n, c}(x)} \quad \forall x \in \mathbb{R}^{p} .
$$

In the formulas, we use non-smoothing weights for the qualitative variable $C$. If there exists a natural ranking of the modalities of $C$ relevant for the regression model, non-smoothing weights can be replaced by smooth ones without affecting the estimators' asymptotic properties, see Bierens (1987) and Delgado and Mora (1995), or our test's validity.

If we overlook the information concerning the splitting as given by the $C_{i}$ 's, we would consider instead the pooling regression model

$$
Y_{i}=r\left(X_{i}\right)+u_{i}, \quad E\left[u_{i} \mid X_{i}\right]=0, \quad i=1, \ldots, n
$$

Thus we will estimate the function $r(\cdot)$ by a kernel estimate on the whole sample given by

$$
r_{n}(x)=\frac{\left(n h^{p}\right)^{-1} \sum_{i=1}^{n} Y_{i} K\left(\frac{x-X_{i}}{h}\right)}{f_{n}(x)}, \quad \text { where } f_{n}(x)=\left(n h^{p}\right)^{-1} \sum_{i=1}^{n} K\left(\frac{x-X_{i}}{h}\right), \quad \forall x \in \mathbb{R}^{p},
$$

and $h$ is a bandwidth parameter converging to zero. These estimators respectively converge to $r(\cdot)$ and $f(\cdot)$, the marginal density of $X$.

The hypothesis of interest is the constancy of the regression function $R(\cdot, C=c)$ for different values of $c$. This means that we are not loosing any information by disregarding the $C_{i}$ 's and estimating the simpler regression Model (2.2) instead of (2.1). Thus the null hypothesis reads

$$
H_{0}: R(X, C)=r(X) \text { a.s. }
$$

This intuitive formulation enables us to deal with the testing problem as a comparison of two nested models, whatever the number of subsamples is. As $H_{0}$ corresponds to the non-significance of the discrete variable $C$, we can built our test statistic in a way similar to Lavergne and Vuong (2000), who test for omitted continuous variables. Let $u \equiv Y-r(X)$. We consider an estimate of $E\left[E^{2}(u \mid X, C) \Psi(X, C)\right]=E\left[(R(X, C)-r(X))^{2} \Psi(X, C)\right]$, which is zero under $H_{0}$ and strictly positive under any alternative to $H_{0}$, for any function $\Psi(\cdot, \cdot)$ such that $\Psi(\cdot, c)$ is strictly positive on the support of $f_{c}(\cdot)$ for any $c$. Because of the form of the kernel estimate, it is convenient

\footnotetext{
${ }^{4}$ For exposition's ease, we do not make explicit the dependence of the bandwidths on the (sub)sample size.
} 
to use $f^{2}(X) f_{C}(X)$ as a weighting function. ${ }^{5}$ This device is analogous to the one used in other semiparametric estimation and testing problems, see e.g. Powell, Stock and Stoker (1989), Fan and $\mathrm{Li}$ (1996), Lavergne and Vuong (2000) and Zheng (1996). If the quantities $u_{i} f\left(X_{i}\right)$ were observed, a sample analog of $E\left[E^{2}(u f(X) \mid X, C) f_{C}(X)\right]=E\left[u f(X) E(u f(X) \mid X, C) f_{C}(X)\right]$ would be

$$
V_{0 n}=\frac{1}{n^{(2)}} \sum_{a} u_{i} u_{j} f\left(X_{i}\right) f\left(X_{j}\right) \frac{1}{h_{C_{i}}^{p}} \tilde{K}_{n i j} w_{n i j}, \text { where } \tilde{K}_{n i j} \equiv\left(1 / h_{C_{i}}^{p}\right) K\left[\frac{X_{i}-X_{j}}{h_{C_{i}}}\right],
$$

$w_{n i j}=\frac{n-1}{n_{C_{i}}-1} \mathbb{I}\left[C_{i}=C_{j}\right], \sum_{a}$ denotes summation over the arrangements of $m$ distinct elements $\left\{i_{1}, \ldots, i_{m}\right\}$ from $\{1, \ldots, n\}$, and $n^{(m)}=n ! /(n-m)$ ! is the number of these arrangements. Now, because we do not know the $u_{i}$ 's and $f\left(X_{i}\right)^{\text {'s, }}$, we replace them by their kernel estimates, and we drop equal indices in the final sum. ${ }^{6}$ We then obtain the statistic

$$
V_{n}=\frac{1}{n^{(4)}} \sum_{a}\left(Y_{i}-Y_{k}\right)\left(Y_{j}-Y_{l}\right) K_{n i k} K_{n j l} \tilde{K}_{n i j} w_{n i j}, \text { where } K_{n i j} \equiv\left(1 / h^{p}\right) K\left[\frac{X_{i}-X_{j}}{h}\right] .
$$

\subsection{Asymptotic behavior of $V_{n}$}

Theorem 1 gives the behavior of $V_{n}$ under the set of hypotheses

$$
H_{1 n}: \quad R(X, C)=r(X)+\delta_{n} d(X, C), \delta_{n} \in[0,1]
$$

A fixed alternative corresponds to $\delta_{n}=1 \forall n$, while the null hypothesis corresponds to $\delta_{n}=0 \forall n$. ${ }^{7}$ This general formulation allows to deal with some local alternatives whose rates of convergence to $H_{0}$ are given by the rate of decrease of $\delta_{n}$ to zero.

We call $\mathcal{U}^{p}$ the class of integrable uniformly continuous functions from $\mathbb{R}^{p}$ to $\mathbb{R}$, and $\mathcal{D}_{m, q}^{p}$ the class of $m$-times differentiable functions from $\mathbb{I R}^{p}$ to $I R$ with derivatives of order $m$ that are uniformly Lipschitz continuous of order $q, q \in[0,1)$. For $m \geq 2$, we define $\mathcal{K}_{m}^{p}$ as the class of integrable functions $K(\cdot)$ from $\mathbb{R}^{p}$ to $\mathbb{R}$ with compact support, satisfying $\int K(s) d s=1$ and

$$
\int s_{1}^{\alpha_{1}} \ldots s_{p}^{\alpha_{p}} K(s) d s=0 \quad \text { for } \alpha_{i} \in\{0,1, \ldots, m-1\}, \forall i=1, \ldots, p, \quad 0<\sum_{i=1}^{p} \alpha_{i} \leq m-1 .^{8}
$$

\footnotetext{
${ }^{5}$ We could also choose $f^{2}(X) f_{C}(X) v(X), v(\cdot)$ being a known function, used for instance to restrict attention to a particular interval. Our results can be easily extended to this formulation.

${ }^{6}$ For significance testing of continuous variables, Lavergne and Vuong (2000) show that dropping equal indices does not change the asymptotic distribution of their statistic but reduces its small-sample bias. In our case, dropping equal indices is essential to get an asymptotically unbiased statistic under $H_{0}$.

${ }^{7}$ We let $d(X, C) \equiv 0$ if $\delta_{n}=0$.

${ }^{8}$ It suffices that $\int K(s) d s>0$ to obtain a consistent test, but the unity integral assumption is not restrictive.
} 
We let $\sigma_{C}^{2}(X) \equiv E\left[u^{2} \mid X, C\right]=E\left[(Y-r(X))^{2} \mid X, C\right]$ and we label it the "conditional variance" (with respect to both $X$ and $C$ ) from Model (2.2). We also let $w_{C C^{\prime}} \equiv \frac{1}{p_{C}} \mathbb{I}\left[C=C^{\prime}\right]$ and $K_{a}(\cdot) \equiv(1 / a) K(\cdot / a)$. We define $*$ as the convolution operator, i.e. for kernels $K(\cdot)$ and $L(\cdot)$,

$$
(K * L)(s)=\int_{\mathbb{R}^{p}} K(v) L(s-v) d v .
$$

Assumption $2.1\left\{\left(C_{i}, X_{i}, Y_{i}\right), i=1, \ldots, n\right\}$ is an i.i.d. sample from a random variable $(C, X, Y)$ on $\boldsymbol{C} \times \mathbb{R}^{p} \times \mathbb{R}$, where $C$ is a discrete variable on $\boldsymbol{C}=\{1, \ldots, \bar{C}\}$, with corresponding strictly positive probabilities $p_{1}, \ldots, p_{\bar{C}}$, and $E\left(Y^{8}\right)<\infty$.

Assumption 2.2 (i) For each $c=1, \ldots, \bar{C}, f_{c}(\cdot)$ and $R(\cdot, c) f_{c}(\cdot)$ belong to $\mathcal{U}^{p} \cap \mathcal{D}_{m, q}^{p}, m \geq 2$, and also $\sigma_{c}^{2}(\cdot) f_{c}(\cdot)$ belongs to $\mathcal{U}^{p}$. (ii) $K \in \mathcal{K}_{m}^{p}, m \geq 2$.

Our statistic involves one bandwidth $h$ for the pooling null model as well as a set of $\bar{C}$ bandwidths for the alternative general model. We restrict our analysis to the following case.

Assumption 2.3 For $c=1, \ldots, \bar{C}, h_{c} / h \rightarrow a_{c}$ with $0<a_{c}<\infty$ and $n h^{p} \rightarrow+\infty$.

Theorem 1 Under Assumptions 2.1, 2.2 and 2.3, if $n h^{(p / 2)+2(m+q)} \rightarrow 0$, then as $n \rightarrow+\infty$,

$$
\text { (i) } n h^{p / 2} V_{n} \stackrel{d}{\longrightarrow} N\left(A \mu, \omega^{2}\right) \quad \text { if } \delta_{n}^{2} n h^{p / 2} \rightarrow A<\infty,
$$$$
\text { (ii) } n h^{p / 2} V_{n} \stackrel{p}{\longrightarrow}+\infty \quad \text { if } \delta_{n}^{2} n h^{p / 2} \rightarrow+\infty \text {, }
$$

where $\mu=E\left[d^{2}(X, C) f^{2}(X) f_{C}(X)\right], \omega^{2}=2 E\left[\sigma_{C}^{2}(X) \sigma_{C^{\prime}}^{2}(X) f^{4}(X) E_{C C^{\prime}}(X)\right]$,

$$
E_{C C^{\prime}}(X)=\int\left[K_{a_{C}}(s) w_{C C^{\prime}}-2\left(K * K_{a_{C}}\right)(s) \frac{f_{C}(X)}{f(X)}+\frac{g^{2}(X, s)}{f^{2}(X)}\right]^{2} d s
$$

and $g^{2}(X, s)=\sum_{c=1}^{\bar{C}} p_{c} f_{c}^{2}(X)\left(K * K * K_{a_{c}}\right)(s)$.

We postpone discussion of our assumptions to the next subsection. As shown in the proofs, the behavior of $V_{n}$ depends upon whether $H_{0}$ holds or does not hold. Under the alternative, $V_{n}$ converges to a normal distribution with the usual $\sqrt{n}$-rate of convergence. But under the null, the $\sqrt{n}$-asymptotic distribution of $V_{n}$ is degenerate. This leads us to consider higher-order terms in the asymptotic expansion of $V_{n}$. Similar situations also arise in parametric specification testing using functional estimation or significance testing of continuous covariates in nonparametric regression. In such procedures, one also compares two nested models with statistics similar to 
$V_{0 n}$, where the unknown elements of the null model are replaced by parametric or nonparametric estimators. Because in the latter cases, the null model has a lower effective dimension than the alternative model, estimators in the former have faster pointwise rates of convergence than estimators in the latter. Thus plugging-in estimators in $V_{0 n}$ does not affect its asymptotic behavior. In contrast, both the general Model (2.1) and the restricted Model (2.2) have the same effective dimension, so that estimators have similar rates of convergence in both models. Consequently, the asymptotic behavior of $V_{n}$ differs from the one of $V_{0 n}$. Nevertheless, our results show that plugging-in estimators influences the asymptotic variance under $H_{0}$, but affects neither the asymptotic expectation nor the rate of convergence.

In writing the asymptotic variance $\omega^{2}$, we have adopted the following conventions:

$$
\begin{gathered}
E\left[\sigma_{C}^{2}(X) \sigma_{C^{\prime}}^{2}(X) \Psi(X)\right] \equiv \sum_{c, c^{\prime}} p_{c} p_{c^{\prime}} \int \sigma_{c}^{2}(x) \sigma_{c^{\prime}}^{2}(x) \Psi(x) f_{c}(x) f_{c^{\prime}}(x) d x \\
E\left[\sigma_{C}^{2}(X) \sigma_{C^{\prime}}^{2}(X) \Psi(X) \mathbb{I}\left[C=C^{\prime}\right]\right] \equiv \sum_{c, c^{\prime}} p_{c} p_{c^{\prime}} \int \sigma_{c}^{2}(x) \sigma_{c^{\prime}}^{2}(x) \Psi(x) f_{c}(x) f_{c^{\prime}}(x) \mathbb{I}\left[c=c^{\prime}\right] d x .(2.4)
\end{gathered}
$$

The asymptotic variance of $V_{n}$ under $H_{0}$ has a quite complicated form. ${ }^{9}$ First, it depends on the cross-products between $\sigma_{c}^{2}(\cdot)$ and $\sigma_{c^{\prime}}^{2}(\cdot)$ for different $c$ and $c^{\prime}$, see Härdle and Marron (1990) for a similar feature. Second, it explicitely depends on the difference in designs between subsamples, through the ratios $f_{c}(\cdot) / f(\cdot)$ and $g^{2}(\cdot, \cdot) / f^{2}(\cdot)$. The first quantity is the ratio of the conditional density of $X$ given $C=c$ to the "average" marginal density $f(\cdot)=\sum_{c=1}^{\bar{C}} p_{c} f_{c}(\cdot)$. To interpret the second quantity, assume that $a_{c}=a$ for any $c$. Then $g^{2}(\cdot, s) / f^{2}(\cdot)=G^{2}(\cdot) / f^{2}(\cdot)\left(K * K * K_{a}\right)(s)$, where $G^{2}(\cdot)=\left(\sum_{c=1}^{\bar{C}} p_{c} f_{c}^{2}(\cdot)\right)$. Thus the second ratio can be given the interpretation of a "normalized variance" of $f_{c}(\cdot)$. When $X$ is independent of $C$, both $f_{c}(\cdot) / f(\cdot)$ and $G^{2}(\cdot) / f^{2}(\cdot)$ equal one for any $x$ and $c$. As we do not require independence, the designs can markedly differ across subsamples and, in general, the latter ratios introduce different weights across the subsamples and the values of explanatory variables. Therefore, it seems impossible to find a kernel that would minimize the variance irrespective of the regressors' designs.

\footnotetext{
${ }^{9}$ It is written in a way that suggests an estimator similar to $V_{n}$. See the Appendix for an extensive form of $\omega^{2}$.
} 


\subsection{Discussion on the assumptions and bandwidths' choice}

Assumption 2.1 allows for any dependence between $(X, Y)$ and $C .{ }^{10}$ Hence, the residuals' distribution is not restricted to be identical for different values of $C$. The residuals can also be conditionally heteroskedastic. Assumption 2.2 requires smoothness conditions on the underlying functions and kernels that are standard in nonparametric estimation. The compactness of the support of $K(\cdot)$ could be relaxed, but this would lead to very tedious proofs. Our assumptions on the bandwidth $h$ include the usual ones, and specifically imply that $h \rightarrow 0$ as $n \rightarrow \infty$, while its rate of decrease is restricted by $n h^{p} \rightarrow+\infty$. The condition $n h^{(p / 2)+2(m+q)} \rightarrow 0$ relates the rate of convergence of the statistic and its bias rate. When comparing two univariate nonparametric regression curves, Härdle and Marron (1990) obtain a statistic with a bias of order $(1 / n h)$, which needs to be estimated to construct a test. ${ }^{11}$ In our case, the leading term that determines the asymptotic distribution of $V_{n}$ is unbiased under the null hypothesis. A bias of order $h^{2(m+q)}$ appears in the remaining terms because of our ignorance of the true function $r(\cdot)$. This bias is controlled through the aforementioned condition. With respect to the optimal rate for estimation of the regression function, i.e. $h \propto n^{-1 /[p+2(m+q)]}$, this condition implies undersmoothing as is usual in semiparametric estimation, see Robinson (1988) and Powell, Stock and Stoker (1989) among others. ${ }^{12}$ Finally, note that our conditions implicitely require $m>p / 4$.

Assumption 2.3 ensures on the one hand that each bandwidth $h_{c}$ converges to zero at a similar rate and on the other hand that the bandwidth $h$ for the pooling model decreases at the same rate. This allows for dependence of the bandwidths on the (sub)sample sizes. The first condition is imposed in all work on the topic using smoothing techniques and is even strengthened to equal bandwidths across subsamples in most papers, see the Introduction for references. The second condition is also imposed in Young and Bowman (1995), where a pooled nonparametric regression is compared to nonparametric regressions based upon subsamples. These authors further argue for equal bandwidths when the design densities are identical across subsamples, so as to equate estimation biases in all models under $H_{0}$. By contrast, Baltagi, Hidalgo and Li (1996) assume oversmoothing of the pooling model by imposing $h_{c} / h^{2}=o(1)$.

\footnotetext{
${ }^{10}$ That is, it allows for $\operatorname{Pr}[(X, Y) \leq(x, y), C=c] \neq \operatorname{Pr}\left[(X, Y) \leq(x, y), C=c^{\prime}\right]$ for $c \neq c^{\prime}$. In particular, the distribution of the regressors can vary across subsamples. See the end of Section 3.1 for a discussion on this point.

${ }^{11} \mathrm{~A}$ similar feature would appear if we would have not dropped equal indices in $V_{n}$.

${ }^{12}$ However, the optimal bandwidth for testing is likely to be different from the optimal bandwidth for estimation, see Guerre and Lavergne (1999) for the case of nonparametric specification testing.
} 
Why is it sensible to use similar amounts of smoothing for all estimators? From an estimation viewpoint, there is no reason why we should employ different smoothing rates in each model. Indeed, a discrete variable does not affect the rate of convergence of nonparametric estimators and does not create any bias in estimation, see e.g. Bierens (1987) and Delgado and Mora (1995). Moreover, each subsample size increases at the same rate as the whole sample size. Therefore, if one wants to select the parameters with respect to some optimality measure, the resulting bandwidths, while depending on possibly different unknown constants, should asymptotically follow the same rate of decrease to zero. ${ }^{13}$

From a testing viewpoint, using bandwidths that fulfil Assumption 2.3 seems the easiest way to put models on equal footing in the testing procedure, because the regressors' dimension is the same between models. For a more theoretical justification, let us consider what happens if we use different amounts of smoothing in the competing models. In this aim, we introduce a new bandwidth $a$ for the pooling model and consider the statistic

$$
V_{n}=\frac{1}{n^{(4)}} \sum_{a}\left(Y_{i}-Y_{k}\right)\left(Y_{j}-Y_{l}\right) \frac{1}{a^{2 p}} K\left(\frac{X_{i}-X_{k}}{a}\right) K\left(\frac{X_{j}-X_{l}}{a}\right) \tilde{K}_{n i j} w_{n i j}
$$

We maintain Assumption 2.3, which now only imposes similar rates of convergence for the $h_{c}$ 's. If we assume oversmoothing in the null model with respect to the general one by imposing $h / a=o(1), V_{n}$ is asymptotically equivalent to $V_{0 n}$ and has a $n h^{p / 2}$ rate of convergence. ${ }^{14}$ Conversely, if we assume that $a / h=o(1)$, we obtain a rate of convergence of $n a^{p / 2}$ for $V_{n} \cdot{ }^{15}$ These results, together with Theorem 1 under the assumption $a=h$, gives us a rate of convergence for $V_{n}$ of $\min \left(n h^{p / 2}, n a^{p / 2}\right)$. To get the most powerful test, we should clearly maximize this rate by equating $h$ and $a$, that is, by choosing similar amount of smoothing in the competing models. ${ }^{16}$ Hence Assumption 2.3 allows our statistic to be as efficient as possible and in this sense is not restrictive. From a practical viewpoint, our formal analysis under Assumption 2.3 leads to an approximation that is likely to be more useful than a result obtained under one of the alternative assumptions $a / h=o(1)$ or $h / a=o(1)$. Each alternative assumption is akin to ignore some interaction terms in the variance of the statistic, which are however present because these

\footnotetext{
${ }^{13}$ We are also using the same kernel for all estimators because, from their definitions, both functions $r(\cdot)$ and $R(\cdot, \cdot)$ have similar smoothness properties. Hence the order of the kernel should be the same for both models. However, this restriction is not crucial, and a more general result could account for different kernels.

${ }^{14}$ This easily follows by adapting our proof. See Lavergne and Vuong (2000) for a related result.

${ }^{15}$ This also follows by adapting our proof.

${ }^{16}$ The supplementary restrictions on the bandwidths are similar across cases.
} 
ratios are never zero in practice. By contrast, our approach explicitely takes these interaction terms into account.

\section{$2.4 \quad$ Testing procedure and extensions}

From a reasoning analogous to the one leading to (2.3), the variance $\omega^{2}$ can be estimated as

$$
\omega_{n}^{2}=\frac{2}{n^{(6)}} \sum_{a}\left(Y_{i}-Y_{k}\right)\left(Y_{i}-Y_{k^{\prime}}\right)\left(Y_{j}-Y_{l}\right)\left(Y_{j}-Y_{l^{\prime}}\right) K_{n i k} K_{n i k^{\prime}} K_{n j l} K_{n j l^{\prime}} K_{n i j} E_{n i j},
$$

where $E_{n i j}=\mathbb{I}\left[f_{n}\left(X_{i}\right) \geq b_{n}\right] \int\left[K(s) w_{n i j}-2(K * K)(s) \frac{f_{n, C_{i}}\left(X_{i}\right)}{f_{n}\left(X_{i}\right)}+\frac{g_{n}^{2}\left(X_{i}, s\right)}{f_{n}^{2}\left(X_{i}\right)}\right]^{2} d s$,

$g_{n}^{2}(x, s)=\sum_{c=1}^{\bar{C}}\left(n_{c} / n\right) f_{n, c}^{2}(x)\left(K * K * K_{a_{c}}\right)(s), \forall x \in \mathbb{R}^{p}$, and $b_{n}$ is a trimming parameter such that $b_{n}=o(1)$. This trimming is necessary to get rid of edge effects, that is, to avoid undue influence from observation points where the density $f(\cdot)$ is small. ${ }^{17}$ An alternative estimator, which is computationally less demanding but more biased in small samples, is

$$
\omega_{n}^{2}=\frac{2}{n^{(2)}} \sum_{a} u_{n i}^{2} f_{n i}^{2} u_{n j}^{2} f_{n i}^{2} \tilde{K}_{n i j} E_{n i j}, \quad \text { where } \quad u_{n i} f_{n i} \equiv\left[Y_{i}-r_{n}\left(X_{i}\right)\right] f_{n}\left(X_{i}\right) .
$$

Theorem 2 Under Assumptions 2.1, 2.2 and 2.3, if $\left(b_{n} \sqrt{n} h_{n}^{p}\right)^{-1} \rightarrow 0, b_{n}^{-1} h^{m+q} \rightarrow 0$ and $n h^{(p / 2)+2(m+q)} \rightarrow 0$, then as $n \rightarrow+\infty$,

$$
\begin{array}{lll}
\text { (i) } n h^{p / 2} V_{n} / \omega_{n} \stackrel{d}{\longrightarrow} N(A \mu / \omega, 1) & \text { if } \delta_{n}^{2} n h^{p / 2} \rightarrow A<\infty, \\
\text { (ii) } n h^{p / 2} V_{n} / \omega_{n} \stackrel{p}{\longrightarrow}+\infty & \text { if } \delta_{n}^{2} n h^{p / 2} \rightarrow+\infty .
\end{array}
$$

Theorem 2 provides an equality test across nonparametric regressions. By letting $\delta_{n}=0$, the test statistic is asymptotically $N(0,1)$ under $H_{0}$ and, by letting $\delta_{n}=1$, it diverges to $+\infty$ under any fixed alternative to $H_{0}$. The resulting test is therefore a one-sided normal test and is consistent against any fixed alternative. In addition, the test has power to detect local alternatives of the type $H_{1 n}$ provided that $\delta_{n}^{2} n h^{p / 2} \rightarrow+\infty$. In practice, $V_{n}$ can be more easily computed through

$$
\begin{aligned}
n^{(4)} V_{n}= & \sum_{a} u_{n i} f_{n i} u_{n j} f_{n j} \tilde{K}_{n i j} w_{n i j}+2 \sum_{a} u_{n i} f_{n i}\left(Y_{i}-Y_{j}\right) K_{n i j} \tilde{K}_{n i j} w_{n i j} \\
& -\sum_{a}\left(Y_{i}-Y_{j}\right)^{2} K_{n i j}^{2} \tilde{K}_{n i j} w_{n i j}-\sum_{a}\left(Y_{i}-Y_{k}\right)\left(Y_{j}-Y_{k}\right) K_{n i k} K_{n j k} \tilde{K}_{n i j} w_{n i j} .
\end{aligned}
$$

Different extensions of the procedure can be proposed. First, the procedure can be applied to test the significance of any set of qualitative variables in a nonparametric regression. The

\footnotetext{
${ }^{17}$ Unreported simulation results indicate that trimming, though necessary in theory, is not crucial in practice.
} 
variable $C$ is used to recover any combination of the values of the initial discrete variables. Second, one can easily introduce discrete variables in the regressors that are not under test. That is, we can consider $(X, D)$ instead of $X$, where $D$ is a set of discrete covariates. In that case, one should introduce $D$ in the different functions considered, so that $r(X)$ becomes $r(X, D)$, ... The asymptotic properties of $V_{n}$ are as in Theorem 1. As noted before, we can equivalently use either smooth or non-smoothing weights for the discrete variables in $D$ without affecting our procedure's properties. Third, though the theory is developped for generic bandwidths, our result can be extended to vanishing individual bandwidths for each univariate regressor. Fourth, as suggested by a referee, we can allow for data-driven bandwidths.

Theorem 3 If $K(\cdot)$ is differentiable with bounded partial derivatives, Theorem 2 extends to the case of random data-driven $\widehat{h}$ and $\widehat{h}_{c}, c=1, \ldots, \bar{C}$, if there exist deterministic sequences $h$ and $h_{c}, c=1, \ldots, \bar{C}$, that fulfil the assumptions of Theorem 2 such that $(\widehat{h}-h) / h$ and $\left(\widehat{h}_{c}-h_{c}\right) / h_{c}, c=1, \ldots, \bar{C}$, are all $o_{p}(1)$.

Fifth, as detailled in the next section, both the independence assumption and the identical distribution assumption can be relaxed to some extent.

\section{Case of a fixed $C$}

\subsection{Cross-section data}

There exist situations where the variable defining the division of subsamples is not random, for instance when testing for poolability of cross-section data. This is also true for experiments in which one can control for treatments. The results from the previous section can be adapted to this context. Specifically, let $C$ be a variable taking integer values in $\{1, \ldots, \bar{C}\}$. For each $c$, assume that we have at hand an i.i.d. sample of size $n_{c}$ from a random variable $\left(X_{c}, Y_{c}\right)$ on $\mathbb{R}^{p} \times \mathbb{R}$, such that $X_{c}$ has marginal density $f_{c}(\cdot)$. We employ similar notations as in Section 2 , so that, for each $c$, the sample from $\left(X_{c}, Y_{c}\right)$ is denoted $\left\{\left(X_{i}, Y_{i}\right), i=1+\sum_{c^{\prime}<c} n_{c^{\prime}}, \ldots, \sum_{c^{\prime} \leq c} n_{c^{\prime}}\right\}$ and $\sum_{c=1}^{\bar{C}} n_{c}=n$. We then consider the general regression model

$$
Y_{i}=R\left(X_{i}, c\right)+U_{i}, \quad E\left[U_{i} \mid X_{i}\right]=0 \quad i=1, \ldots, n,
$$

so that now $R(\cdot, c)$ denotes the regression function of $Y_{c}$ on $X_{c}$. For each $c$, nonparametric kernel estimators of $R(\cdot, c)$ and $f_{c}(\cdot)$ are defined as in the previous section. Overlooking the 
information given by $C$ and assuming falsely that the observations constitute an i.i.d. sample leads to consider the pooling regression model (2.2). Nonparametric kernel estimators of $f_{n}(\cdot)$ and $r_{n}(\cdot)$ are defined as in the previous section, but their interpretation radically changes. Here $f_{n}(\cdot)$ estimates $f(\cdot)=\sum_{c=1}^{\bar{C}} n_{c} f_{c}(\cdot) / n$, which is not the marginal density of an observed random variable $X$, but the density of a hypothetical variable constructed from the different $X_{c}$ 's. Similarly, $r_{n}(\cdot)$ estimates $r(\cdot)=\sum_{c=1}^{\bar{C}} n_{c} R(\cdot, c) f_{c}(\cdot) / n f(\cdot)$, which is no more a conditional expectation function, but a weighted average of the regression functions $R(\cdot, c), c=1, \ldots, \bar{C}$.

While the two models are now interpreted differently, the framework is similar. The null hypothesis of interest is still the constancy of the regression function $R(\cdot, c)$ for different values of $c$, so that it is possible to estimate it from the pooling model, even though the densities $f_{c}(\cdot)$ differ for different values of $c$. Thus, the null hypothesis of interest reads

$$
H_{0}: R(X, c)=r(X) \text { a.s. } \quad \forall c=1, \ldots, \bar{C}
$$

and the statistic $V_{n}$ is constructed as in $(2.3)$. We let $\sigma_{c}^{2}(X) \equiv E\left[\left(Y_{c}-r\left(X_{c}\right)\right)^{2} \mid X_{c}=X\right]$. The following assumption summarizes the considered setup for a fixed $C$.

Assumption 3.1 Let $C$ be a fixed variable taking integer values in $\{1, \ldots, \bar{C}\}$. For $c=1, \ldots, \bar{C}$, each subsample $\left\{\left(X_{i}, Y_{i}\right), i=1+\sum_{c^{\prime}<c} n_{c^{\prime}}, \ldots, \sum_{c^{\prime} \leq c} n_{c^{\prime}}\right\}$ is an i.i.d. sample of size $n_{c}$ from a random variable $\left(X_{c}, Y_{c}\right)$ on $\mathbb{R}^{p} \times \mathbb{R}$, such that $X_{c}$ has marginal density $f_{c}(\cdot)$ and $E\left(Y_{c}^{8}\right)<\infty$. The subsamples are independent. For any $c, n_{c} / n \rightarrow p_{c}$ as $n=\sum_{c=1}^{\bar{C}} n_{c} \rightarrow+\infty$.

Theorem 4 Under Assumptions 3.1, 2.2 and 2.3, if $n h^{p / 2+2(m+q)} \rightarrow 0$, then as $n \rightarrow+\infty$,

$$
\begin{array}{lll}
\text { (i) } n h^{p / 2} V_{n} \stackrel{d}{\longrightarrow} N\left(A \mu, \omega^{2}\right) & \text { if } \delta_{n}^{2} n h^{p / 2} \rightarrow A<\infty, \\
\text { (ii) } n h^{p / 2} V_{n} \stackrel{p}{\longrightarrow}+\infty & \text { if } \delta_{n}^{2} n h^{p / 2} \rightarrow+\infty,
\end{array}
$$

where $\mu=\sum_{c=1}^{\bar{C}} p_{c} E\left[d^{2}(X, c) f^{2}(X) f_{c}(X)\right], \omega^{2}=2 \sum_{c, c^{\prime}} p_{c} p_{c^{\prime}} E\left[\sigma_{c}^{2}(X) \sigma_{c^{\prime}}^{2}(X) f^{4}(X) E_{c c^{\prime}}(X)\right]$,

$$
E_{c c^{\prime}}(X)=\int\left[K_{a_{c}}(s) w_{c c^{\prime}}-2\left(K * K_{a_{c}}\right)(s) \frac{f_{c}(X)}{f(X)}+\frac{g^{2}(X, s)}{f^{2}(X)}\right]^{2} d s,
$$

$w_{c c^{\prime}}=\left(1 / p_{c}\right) \mathbb{I}\left[c=c^{\prime}\right]$ and $g^{2}(X, s)=\sum_{c=1}^{\bar{C}} p_{c} f_{c}^{2}(X)\left(K * K * K_{a_{c}}\right)(s) .{ }^{18}$

Compared to the previous section, we have relaxed the assumption of identically distributed data across subsamples, but still assume independence across subsamples, which is typically

\footnotetext{
${ }^{18}$ In writing $\omega^{2}$, we use similar conventions as in (2.4).
} 
the case for cross-section data. The proof of Theorem 4 mainly follows the one of Theorem 1 , see Appendix A for some brief explanations. Theorems 2 and 3 can be similarly extended. A testing procedure can then be based on $n h^{p / 2} V_{n} / \omega_{n}$, where $\omega_{n}$ is an estimator of the asymptotic variance $\omega^{2}$ analogous to the ones in Section 2.4. As before, the test is a one-sided normal test, is consistent against any alternative and detects local alternatives of the type $H_{1 n}$ approaching the null at a rate slower than $\left(n h^{p / 2}\right)^{-1 / 2} \cdot{ }^{19}$

There are interesting connections between our procedure and analysis of covariance. The simple analysis of variance model reads

$$
Y_{i}=\beta_{C_{i}}+U_{i}, \quad E\left[U_{i}\right]=0
$$

For testing the hypothesis $\beta_{c}=\beta$, for all $c$, the usual testing procedure is built upon

$$
S=(1 / n) \sum_{c} n_{c}\left[\bar{Y}_{c}-\bar{Y}\right]^{2},
$$

where $\bar{Y}_{c}=\left(1 / n_{c}\right) \sum_{i=1}^{n} Y_{i} \mathbb{I}\left[C_{i}=c\right]$ and $\bar{Y}=(1 / n) \sum_{i=1}^{n} Y_{i}$. Now $S$ equivalently reads

$$
S=\left(1 / n^{4}\right) \sum_{i, j, k, l}\left(Y_{i}-Y_{k}\right)\left(Y_{j}-Y_{l}\right) \frac{n}{n_{C_{i}}} \mathbb{I}\left[C_{i}=C_{j}\right]
$$

Our statistic $V_{n}$ is analogous to $S$ (with the slight difference that it excludes equal indices in the sum), but weights the first differences in the dependent variable by quantities that depend upon explanatory variables. Thus our testing procedure provides a nonparametric analysis of covariance, which allows to detect any differential effect of the regressors on the dependent variable across the considered subsamples without imposing any parametric assumption.

Three main remarks follow from this interpretation. The first remark is related to the choice of weights in the null hypothesis

$$
H_{0}: R(X, c)=r(X) \equiv \frac{\sum_{c=1}^{\bar{C}} p_{c} R(X, c) f_{c}(X)}{\sum_{c=1}^{\bar{C}} p_{c} f_{c}(X)} \text { a.s. } \forall c=1, \ldots, \bar{C} .
$$

When the observations constitute a unique random sample, it is meaningful to use a weighting scheme proportional to $p_{c} f_{c}($.$) . When C$ is fixed, the use of "frequencies" as weights in $H_{0}$ is no longer readily interpretable. Obviously, there exist other equivalent formulations of the

\footnotetext{
${ }^{19}$ By contrast, Yatchew's (1999) test can detect local alternatives that converges to the null at a rate slower than $n^{-1 / 4}$, which is in turn slower than $\left(n h^{p / 2}\right)^{-1 / 2}$.
} 
hypothesis that $R(\cdot, c)$ is constant in $c$ that use different weighting schemes. To each formulation corresponds a test statistic, whose relative merits generally depend on the data at hand.

The second remark concerns problems in application and interpretation of the procedure. As noted by Scheffé (1959, p. 198), "it is sometimes said that the analysis of covariance is valid only if the treatments do not affect the values of the concomitant variables. The dictum that the analysis of covariance can be used only in this case would thus confine it to a very restricted situation. The analysis of covariance can be applied to get tests of hypotheses that have correct significance level, but the sense of using these tests must be considered separately in each application." This statement remains true for the nonparametric analysis of covariance proposed here. Specifically, we allow the regressors' designs to vary across subsamples, so that the "treatments" - i.e. the discrete variable $C$ - may affect explanatory variables. The procedure is widely applicable, but may give a right answer to a wrong question. If some of the regressors are "part of the treatment", e.g. if the regressors have different supports depending on the values of $C$, then the null hypothesis $H_{0}$ is no longer meaningful. ${ }^{20}$ This second remark is equally valid in the case of a random $C$.

Third, our procedure only applies for testing the strict equality of regression functions. If one wants to test equality up to some parametric transformations, one should build a specific test statistic that accounts for this at the outset, as done by Härdle and Marron (1990). Even in the simple case of testing for parallelism of the regression curves, which is easily entertained within the linear parametric analysis of covariance framework, adapting our test is not completely straightforward. This extension is left for future work.

\subsection{Panel data}

A useful extension of our test is testing for poolability of panel data. Consider the model

$$
Y_{i t}=R_{t}\left(X_{i t}\right)+U_{i t}, \quad i=1, \ldots, n_{0}, \quad t=1, \ldots, T
$$

At each period $t,\left\{X_{i t}, i=1, \ldots, n_{0}\right\}$ is an i.i.d. sample from $X_{t}$ with density $f_{t}(\cdot)$. The number $T$ of time periods is fixed while the sample size for each period $n_{0}$ goes to infinity. This is the standard case, which is of most practical relevance, since one usually has a large number of

\footnotetext{
${ }^{20}$ In Quade (1982), the first proposed method is valid only under the assumption that the distribution of $X$ does not vary conditionally to $C$. The second one does not require such an assumption and is close in spirit to our analysis, with the major difference that the bandwidth is held fixed.
} 
cross-section units but data covering only a few time-periods. ${ }^{21}$ The null hypothesis of interest is the constancy of the regression function $R_{t}(\cdot)$ over time, that is

$$
H_{0}: R_{t}(X)=r(X) \text { a.s. }
$$

where $r(\cdot) \equiv(1 / T) \sum_{t=1}^{T} f_{t}(\cdot) R_{t}(\cdot) / f(\cdot)$ and $f(\cdot) \equiv(1 / T) \sum_{t=1}^{T} f_{t}(\cdot)$. The statistic $V_{n}$ is

$$
V_{n}=\frac{T}{n^{(4)}} \sum_{a}\left(Y_{i t}-Y_{k r}\right)\left(Y_{j t}-Y_{l s}\right) \frac{1}{h^{2 p}} K\left(\frac{X_{i t}-X_{k r}}{h}\right) \frac{1}{h_{t}^{p}} K\left(\frac{X_{j t}-X_{l s}}{h}\right) K\left(\frac{X_{i t}-X_{j t}}{h_{t}}\right) .
$$

Here $n=n_{0} T, \sum_{a}$ denotes summation over arrangements of distinct indices $\{i t, j t, k r, l s\}, h$ and $h_{t}, t=1, \ldots, T$, are bandwidth parameters such that $h_{t} / h \rightarrow a_{t}, 0<a_{t}<+\infty$, for all $t$. The results of the Section 3.1, where we imposed independence across subsamples, do not readily apply in this context. Nevertheless, as we argue below, this assumption can be weakened and the asymptotic behavior of $V_{n}$ as $n_{0} \rightarrow \infty$ is given by Theorem $4 .{ }^{22}$ Its asymptotic variance is

$$
\omega^{2}=\left(2 / T^{2}\right) \sum_{t=1}^{T} \sum_{t^{\prime}=1}^{T} E\left[\sigma_{t}^{2}(X) \sigma_{t^{\prime}}^{2}(X) f^{4}(X) E_{t t^{\prime}}(X)\right]
$$

where $E_{t t^{\prime}}=\int\left[T K_{a_{t}}(s) \mathbb{I}\left[t=t^{\prime}\right]-2\left(K * K_{a_{t}}\right)(s) \frac{f_{t}(X)}{f(X)}+\frac{g^{2}(X, s)}{f^{2}(X)}\right]^{2} d s$

and $g(X, s)=(1 / T) \sum_{t=1}^{T} f_{t}^{2}(\cdot)\left(K * K * K_{a_{t}}\right)(s)$.

The usual way of considering panel data models in econometrics is to see $R_{t}(\cdot)$ as the conditional expectation of $Y_{t}$ given all past explanatory variables $\left\{X_{1}, \ldots, X_{t}\right\}$ and a timeindependent latent variable $l$. This formulation is quite general, and in particular allows for some lagged dependent variable in the regressors, so that further restrictions are usually imposed on the model. Chamberlain (1984) distinguishes two main restrictions: lack of residual serial correlation and no structural lagged dependent variables.

There is residual serial correlation conditional on a latent variable $l$ if $Y_{t}$ is not independent of $\left\{Y_{1}, \ldots, Y_{t-1}\right\}$ conditional on $\left\{X_{1}, \ldots, X_{t}, l\right\}$.

The relationship of $X$ to $Y$ is static conditional on a latent variable $l$ if $X$ is strictly exogeneous conditional on $l$ and if $Y_{t}$ is independent of $\left\{X_{1}, \ldots, X_{t-1}\right\}$ conditional on $X_{t}$ and $l$. If the

\footnotetext{
${ }^{21}$ The increasing availability of micropanels in which $T$ is not negligible relative to $n_{0}$ motivates rising interest for further analysis. See e.g. Alvarez and Arellano (1998) for recent results and an account of issues that still need to be addressed in a parametric context.

${ }^{22} \mathrm{~A}$ brief account of the necessary adaptations of the proof is given in Appendix A.
} 
relationship of $X$ to $Y$ is static conditional on a latent variable $l$, then there are no structural lagged dependent variables.

Our analysis imposes the two restrictions of no serial residual correlation and of a static relationship of $X$ to $Y$ (both conditional on a latent variable $l$ ). First, though Assumption 3.1 imposes independence between subsamples, inspection of the proofs reveals that we can alleviate the independence requirement and replace it by the assumption of no serial residual correlation. This assumption allows for fixed individual effects correlated with the regressors. In a nonparametric context, such effects are not separately identifiable. ${ }^{23}$ Second, Model (3.2) also assumes that the regression function does not depend on $\left\{X_{1}, \ldots, X_{t-1}\right\}$. This is true when the relationship of $X$ to $Y$ is static conditional on a latent variable $l$. But, as shown by Chamberlain (1984), there is no restriction to assume a static conditional relationship in a fully nonparametric context. It is restrictive only when combined with a specific functional form of the distribution. Hence, the restrictions imposed in our analysis are not as stringent as they may appear at first.

Baltagi, Hidalgo and Li (1996) consider a statistic close to ours, with the crucial difference that they use a smoothing parameter $a$ for the pooling model and the same bandwidth $h$ at each period for Model (3.2). They subsequently impose $h / a^{2}=o(1)$. As a consequence, the asymptotic variance of the test statistic becomes

$$
\omega_{0}^{2}=2 \int K^{2}(s) d s \sum_{t=1}^{T} E\left[\sigma_{t}^{4}(X) f^{4}(X) f_{t}(X)\right] .
$$

Contrary to $\omega^{2}$, the variance $\omega_{0}^{2}$ depends neither on cross-products of conditional variances between periods nor explicitely on the designs' differences between periods. ${ }^{24}$ This occurs because using different amounts of smoothing in the null and the alternative model results in pulling out any cross-effect between periods. This is why the previous authors do not need the assumption of no serial residual correlation. As argued in Section 2.3, the restriction $h / a^{2}=o(1)$ does not allow to attain the maximum rate of convergence of the statistic. The only justification advocated by the authors is that otherwise one should estimate the expectation of $V_{n}$ to recenter the test statistic, and that this would introduce finite-sample bias. This argument is invalid from both a theoretical viewpoint, as our analysis demonstrates, and a practical viewpoint, as shown by the following simulation results.

\footnotetext{
${ }^{23}$ On this topic, see the discussion of Baltagi, Hidalgo and $\mathrm{Li}$ (1996).

${ }^{24}$ Our formula gives the general form of the asymptotic variance $\omega_{0}^{2}$. The one given in Baltagi, Hidalgo and Li (1996) assumes that the density of the regressors remains the same across time and has $f^{5}(\cdot)$ in place of $f^{4}(\cdot) f_{t}(\cdot)$.
} 


\section{Small sample behavior}

We now investigate the behavior of our test through a Monte-Carlo experiment in the case of a random $C$ taking two values 0 or 1 with equal probabilities. We generate the data through

$$
Y=\beta X+\gamma X^{3}+\mathbb{I}[C=0] d(X)+U
$$

where conditional on $C, X$ is generated as $N(C, 1)$ and $U$ is independently distributed as $N(0,1)$. We impose the restriction that $E[d(X) \mid C=0]=0$ and we set parameters $\beta$ and $\gamma$ to -4 and 1 respectively, so that $E(Y \mid C=0)=E(Y \mid C=1)=0$.

The null hypothesis corresponds to $d(X) \equiv 0$. The alternatives are chosen to investigate the power of our test when their magnitude or frequency vary. For the magnitude, we consider three linear alternatives of the form

$$
d(X)=\alpha X
$$

with $\alpha=0.5,1$ and 2 corresponding respectively to $D G P_{1}, D G P_{2}$ and $D G P_{3}$. This allows to compare the performances of our procedure to the standard Chow test based on the true Model (4.1). Alternatives corresponding to varying frequencies are defined through

$$
d(X)=\sin (\alpha \pi X)
$$

with $\alpha=2,1,2 / 3$ and $1 / 2$ corresponding respectively to $D G P_{4}, D G P_{5}, D G P_{6}$ and $D G P_{7}$. These departures from the null are of special interest, for it is known that smooth tests of parametric specification and nonparametric significance tests for continuous regressors are sensitive to the frequency of the alternative, see Hart (1997) and Lavergne and Vuong (2000).

We consider small $(n=100)$ and moderate $(n=200)$ sample sizes and run 2000 replications. Unreported simulation results show that the trimming parameter has very little influence on the results, so that it is arbitrarily set to 0 in all experiments. The bandwidth parameter $h$ is chosen as $h=\hat{s}_{X} n^{-\lambda}$, where $\hat{s}_{X}$ is the estimated standard deviation for all observations of $X$. We let $h_{0}=h_{1}$, so that $a_{0}=a_{1}=a$. This choice allows to save much computations and has also the advantage that it mimics the choice of an uninformed practitioner who only knows that $p_{0}=p_{1}=1 / 2$. For ease of computation, we choose the uniform kernel with support $[-1 / 2,1 / 2]$. Appendix $\mathrm{C}$ gives the values with respect to $a$ of the integrals that enter $\omega^{2} .{ }^{25}$

\footnotetext{
${ }^{25}$ The Mathematica notebook used to compute the integrals can be adapted for other kernels, and is available from the author upon request.
} 
We first focus on how bandwidths' choice for the alternative model, i.e. $h_{0}=h_{1}$, influence the test's outcome, while keeping fixed the restricted model's bandwidth $h$, as done by Lavergne and Vuong (2000) in another context. We then let $a$ vary in the grid $(0.5,1,1.5,2)$, while keeping $h=\widehat{s}_{X} n^{-1 / 5} .^{26}$ Table 1 reports our results for the null hypothesis $\left(D G P_{0}\right)$ and the linear alternatives. For each case, the first row gives the mean of our test statistic with its standard deviation in parentheses. The second row gives empirical rejection rates of the test, the first figure corresponds to a $5 \%$ nominal level and the second one to a $10 \%$ nominal level. The third row gives corresponding size-corrected empirical rejection rates for the alternative, except for $D G P_{0}$ where it gives the empirical critical values at $5 \%$ and $10 \%$ levels. For each sample size, the last row reports rejection rates of the Chow test for the same nominal levels.

The first column of Table 1 relates to the null hypothesis. For any choice of $a$, the test statistic is very close to be unbiased. The standard deviation of our test statistic is close to, but stays smaller than one. This is due partly to the fact that, to save computations, we use the simplest estimator of the variance (2.5), which is positively biased in small samples. A similar feature appears in the simulations performed by Lavergne and Vuong (2000) on their nonparametric significance test for continuous regressors. ${ }^{27}$ While the mean seems to have an inversed U-shape with respect to $a$, no clear relationship appears to link the standard deviation and $a$. Under the null hypothesis, empirical sizes are quite close to nominal levels. There is no evidence of systematic under or overrejection at a $5 \%$ nominal level. At a $10 \%$ level, there is slight underrejection for all our choices of $a$. Finally the empirical critical values are very close to the ones derived from the normal approximation, with for instance a value of 1.645 at a $5 \%$ level when $a=1$ and $n=200$.

Regarding the linear alternatives, we find as expected that power is increasing with the sample size and the magnitude of the departure from the null, as measured by $\alpha$. The choice $a=0.5$ leads to small power, especially for alternatives of little amplitude. Though, our test can reasonably detect moderate linear alternatives for a bandwidth $h_{0}=h_{1}$ at least as great as $h$. Furthermore, for an alternative of amplitude $\alpha=2$, the power performance of our test can equal that of the Chow test, although the design is ideal for the latter. The highest power

\footnotetext{
${ }^{26}$ This choice corresponds to a practical rule-of-thumb and is not supposed to be optimal for the testing problem at hand. In our setup, we have $m=2$ and $q=1$, so that $h$ fulfils Theorem 1 's conditions.

${ }^{27}$ In the latter study, it has also been observed that better estimators of the variance are obtained by using $K^{2}(\cdot)$ instead of $K(\cdot)$ times the integral of $K^{2}(\cdot)$. A similar feature is expected to hold in our case.
} 
is attained for the largest considered bandwidth, which could be expected since the alternative is linear and the kernel smoother is a straight line for large bandwidths. Though, too large a bandwidth is likely to give low power.

Table 2 has the same structure as Table 1 and reports results relative to the sinus alternatives. The empirical power of the test exhibits an inverse U-shape as a function of the bandwidth $h_{0}=h_{1}$ for the high frequency alternative $D G P_{4}$. This suggests that the bandwidth should be adapted to the frequency of the alternative. This is not surprising, as a similar feature appears in other smooth tests, see e.g. the simulations results of Delgado, Dominguez and Lavergne (1998) and Lavergne and Vuong (2000). For comparative purposes, we also provide the empirical rejection rates of the Chow test assuming a linear specification in $X$. The lowest frequency alternative $D G P_{7}$ is close to a linear specification in the range $[-1,1]$. Given that $X$ is $N(0,1)$ when $C=0$, the Chow test therefore performs quite well, while our test has high power for large $a$. For higher frequency alternatives $D G P_{4}$ and $D G P_{5}$, the Chow test has either trivial or low power irrespective of the sample size, while the empirical power of our test can exceed $85 \%$ for a moderate sample size of 200 .

We now focus on how the choice of $h$ influences our test, while keeping fixed the ratio of bandwidths $h_{0} / h=h_{1} / h=1$. We then let $\lambda$ vary in the grid $(1 / 3,1 / 4,1 / 5,1 / 6)$. Table 3 (respectively Table 4) has the same structure as Table 1 (Table 2) and report our results for the null hypothesis and the linear alternatives (respectively the sinus alternatives). For a choice of $\lambda$ of at least $1 / 5$, the statistic is close to be unbiased under $H_{0}$, while its expectation increases when $\lambda$ decreases to $1 / 6$ as expected. The variance is decreasing in $\lambda$. On the whole, decreasing $\lambda$ leads to more rejections. For linear alternatives, empirical power is decreasing in $\lambda$, whether it is size-adjusted or not. For sinus alternatives, empirical power can be low when $\lambda$ is large, especially for a small sample size, while it highly increases when $\lambda$ decreases. For $D G P_{4}$ and $n=100$, size-adjusted power exhibits an inverse U-shape as a function of $h$. In the other cases, it highly increases when $h$ increases.

\section{Conclusion}

In this paper we propose a general test of equality across nonparametric regressions. The procedure is based on the comparison of the regression functions for each subsample with the general one that pools all observations. It applies in a variety of situations, whether the division into 
subsamples is defined in a random way or not. In our presentation, we have first considered the leading situation where a random qualitative variable defines the split into different subsamples. Then, by considering the case where the split depends on a fixed qualitative variable, we have shown how our basic assumptions can be weakened so that our test applies to cross-section and panel data. In summary, our testing procedure is applicable in any case where the observations are i.i.d. within each subsample and where the residuals are uncorrelated across subsamples.

The characteristic feature of our testing problem is that both models (under the null and under the alternative) have the same effective dimension. Hence our procedure uses similar amount of smoothing for the pooling and the alternative model. We have justified this assumption and investigated thoroughly its implications. Though, bandwidths' choice is clearly a key issue for application of the test. Bootstrap methods could be a way to bypass this problem, as they usually provide better approximations to the null distribution than asymptotics do and can be much less sensitive to bandwidths' choice, see e.g. Delgado, Dominguez and Lavergne (1998). This possibility should be investigated from both a theoretical and a practical viewpoint.

\section{Appendix A: Proofs}

In what follows, $u_{i} \equiv Y_{i}-r\left(X_{i}\right), U_{i} \equiv Y_{i}-R\left(X_{i}, C_{i}\right), f_{i} \equiv f\left(X_{i}\right), r_{i} \equiv r_{i}\left(X_{i}\right), d_{i} \equiv d\left(X_{i}, C_{i}\right)$, $\sigma_{i}^{2} \equiv \sigma_{C_{i}}^{2}\left(X_{i}\right)$ and $Z_{i} \equiv\left(C_{i}, X_{i}, Y_{i}\right), i=0,1, \ldots, n$. Also $K_{n i k} \equiv h^{-p} K\left[\left(X_{i}-X_{k}\right) / h\right], h_{i} \equiv h_{n_{C_{i}}}$, $\boldsymbol{K}(\cdot) \equiv|K(\cdot)|$ and $i, j, k, l, i^{\prime}, j^{\prime}, k^{\prime}, l^{\prime}$ refer to indices that are pairwise different unless stated otherwise. We let $\widehat{f}_{i} \equiv(n-1)^{-1} \sum_{k \neq i} K_{n i k}$, and more generally for any index set $I$ not containing $i$ with cardinality $|I|, \widehat{f}_{i}^{I} \equiv(n-1-|I|)^{-1} \sum_{k \neq i, k \notin I} K_{n i k}$. To ease exposition, we assume $h_{c} / h=a_{c}$, for all $c$.

\section{A.1 Proof of Theorem 2.1}

As $Y_{i}-Y_{k}=\left(u_{i}-u_{k}\right)+\left(r_{i}-r_{k}\right)$, and as $K(\cdot)$ is even by Assumption 2.2, we have from (2.3)

$$
\begin{aligned}
V_{n}= & \frac{1}{n^{(4)}} \sum_{a}\left(u_{i}-u_{k}\right)\left(u_{j}-u_{l}\right) K_{n i k} K_{n j l} \tilde{K}_{n i j} w_{n i j}+\frac{2}{n^{(4)}} \sum_{a}\left(u_{i}-u_{k}\right)\left(r_{j}-r_{l}\right) K_{n i k} K_{n j l} \tilde{K}_{n i j} w_{n i j} \\
& +\frac{1}{n^{(4)}} \sum_{a}\left(r_{i}-r_{k}\right)\left(r_{j}-r_{l}\right) K_{n i k} K_{n j l} \tilde{K}_{n i j} w_{n i j}=I_{1}+2 I_{2}+I_{3}, \\
\text { where } I_{1}= & \frac{n-2}{n-3} \frac{1}{n^{(2)}} \sum_{a} u_{i} u_{j} f_{i} f_{j} \tilde{K}_{n i j} w_{n i j}+\frac{2(n-2)}{n-3} \frac{1}{n^{(2)}} \sum_{a} u_{i}\left(\widehat{f}_{i}^{j}-f_{i}\right) u_{j} f_{j} \tilde{K}_{n i j} w_{n i j} \\
& +\frac{n-2}{n-3} \frac{1}{n^{(2)}} \sum_{a} u_{i}\left(\widehat{f}_{i}^{j}-f_{i}\right) u_{j}\left(\widehat{f}_{j}^{i}-f_{j}\right) \tilde{K}_{n i j} w_{n i j}-\frac{2}{n^{(3)}} \sum_{a} u_{i} f_{i} u_{l} K_{n j l} \tilde{K}_{n i j} w_{n i j}
\end{aligned}
$$




$$
\begin{aligned}
- & \frac{2}{n^{(3)}} \sum_{a} u_{i}\left(\widehat{f}_{i}^{j, l}-f_{i}\right) u_{l} K_{n j l} \tilde{K}_{n i j} w_{n i j}+\frac{1}{n^{(4)}} \sum_{a} u_{k} u_{l} K_{n i k} K_{n j l} \tilde{K}_{n i j} w_{n i j} \\
& -\frac{1}{n^{(4)}} \sum_{a} u_{i} u_{j} K_{n i k} K_{n j k} \tilde{K}_{n i j} w_{n i j}=\frac{n-2}{n-3}\left[V_{0 n}+2 I_{1,1}+I_{1,2}\right]-2 I_{1,3}-2 I_{1,4}+I_{1,5}-I_{1,6}, \\
I_{2}= & \frac{1}{n^{(3)}} \sum_{a} u_{i} f_{i}\left(r_{j}-r_{l}\right) K_{n j l} \tilde{K}_{n i j} w_{n i j}+\frac{1}{n^{(3)}} \sum_{a} u_{i}\left(\widehat{f}_{i}^{j, l}-f_{i}\right)\left(r_{j}-r_{l}\right) K_{n j l} \tilde{K}_{n i j} w_{n i j} \\
& -\frac{1}{n^{(4)}} \sum_{a} u_{k}\left(r_{j}-r_{l}\right) K_{n i k} K_{n j l} \tilde{K}_{n i j} w_{n i j}=I_{2,1}+I_{2,2}-I_{2,3} .
\end{aligned}
$$

Propositions 1 to 12 study each of the above terms. Collecting results, it follows that $n h^{p / 2}\left[V_{0 n}-2 I_{1,3}+I_{1,5}\right]=L_{n}+\delta_{n}^{2} n h^{p / 2} \mu_{n}+\delta_{n} \sqrt{n} h^{p / 2} O_{p}(1)+o_{p}(1)$, where $L_{n} \stackrel{d}{\longrightarrow} N\left(0, \omega^{2}\right)$ and $\mu_{n} \rightarrow \mu$ and $n h^{p / 2}\left[V_{n}-\left(V_{0 n}-2 I_{1,3}+I_{1,5}\right)\right]=\delta_{n}^{2} n h^{p / 2} o_{p}(1)+\delta_{n} \sqrt{n} h^{p / 2} O_{p}(1)+\delta_{n} n h^{p / 2} h^{(m+q)} O_{p}(1)+o_{p}(1)$. If $\delta_{n}^{2} n h^{p / 2} \rightarrow A, \delta_{n}^{2} \sqrt{n} h^{p / 2}$ and $\delta_{n} n h^{p / 2} h^{(m+q)}$ are both $o(1)$, so that $n h^{p / 2} V_{n} \stackrel{d}{\longrightarrow} N\left(A \mu, \omega^{2}\right)$. If $\delta_{n}^{2} n h^{p / 2} \rightarrow+\infty, \delta_{n}^{2} \sqrt{n} h^{p / 2}$ and $\delta_{n} n h^{p / 2} h^{(m+q)}$ are both $o\left(\delta_{n}^{2} n h^{p / 2}\right)$, so that $n h^{p / 2} V_{n} \stackrel{p}{\longrightarrow}+\infty$. Q.E.D. In the following proofs, we will rely on the following lemma.

Lemma 1 (Fan and Li, 1996) Let $U_{n}$ be a U-statistic of order $m$ with symmetrical kernel $H_{n}$ such that $E\left[H_{n}\left(Z_{1}, \ldots, Z_{m}\right) \mid Z_{1}\right]=0$ a.s. and $E\left[H_{n}^{2}\left(Z_{1}, \ldots, Z_{m}\right)\right]<\infty$ for each $n$. Let $H_{n, s}=E\left[H_{n}\left(Z_{1}, \ldots, Z_{m}\right) \mid\left(Z_{1}, \ldots, Z_{s}\right)\right], \xi_{s}=\operatorname{Var}\left[H_{n, s}\left(Z_{1}, \ldots, Z_{s}\right)\right]$ for $s=2, \ldots, m$ and $G_{n}\left(Z_{1}, Z_{2}\right)=$ $E\left[H_{n, 2}\left(Z_{1}, Z_{0}\right), H_{n, 2}\left(Z_{2}, Z_{0}\right) \mid\left(Z_{1}, Z_{2}\right)\right]$. If $\xi_{s} / \xi_{2}=o\left(n^{s-2}\right)$ for $s=1, \ldots, m$, and

$$
\frac{E\left[G_{n}^{2}\left(Z_{1}, Z_{2}\right)\right]+n^{-1} E\left[H_{n, 2}^{4}\left(Z_{1}, Z_{2}\right)\right]}{E^{2}\left[H_{n, 2}^{2}\left(Z_{1}, Z_{2}\right)\right]} \rightarrow 0 \quad \text { as } n \rightarrow \infty,
$$

then $n U_{n}$ is asymptotically normal with zero mean and variance $(1 / 2)\left(m^{(2)}\right)^{2} \xi_{2}$.

As we consider U-statistics with non-symmetric kernel in our proofs, we briefly explain how Lemma 1 extends to this case. One first needs to replace $H_{n}\left(Z_{1}, \ldots, Z_{m}\right)$ by the symmetric kernel

$$
\tilde{H}_{n}\left(Z_{1}, \ldots, Z_{m}\right)=\frac{1}{m !} \sum_{p} H_{n}\left(Z_{i_{1}}, \ldots, Z_{i_{m}}\right)
$$

where $\sum_{p}$ denotes summation over the $m$ ! permutations of $(1, \ldots, m)$. If $E\left[\tilde{H}_{n}\left(Z_{1}, \ldots, Z_{m}\right) \mid Z_{1}\right]=0$ a.s., the U-statistic is degenerate and under the assumptions of Lemma 1 converges in distribution to a centered normal distribution. Its asymptotic variance is given by

$$
(1 / 2)\left(m^{(2)}\right)^{2} \xi_{2}=(1 / 2)\left(m^{(2)}\right)^{2}(1 / m !)^{2} \sum_{p} \sum_{p^{\prime}} E\left[H_{n}\left(Z_{i_{1}}, \ldots, Z_{i_{m}}\right) H_{n}\left(Z_{i_{1}^{\prime}}, \ldots, Z_{i_{m}^{\prime}}\right) \mid Z_{1}, Z_{2}\right] .
$$

Hence one needs to determine all the terms in the double summation. Similar expressions are derived for $\xi_{s}, s=3, \ldots, m$. An analogous result also holds for two U-statistics of order $m_{1}$ and $m_{2}$ with respective kernels $H_{1, n}$ and $H_{2, n}$. Their asymptotic covariance is then given by

$$
(1 / 2) m_{1}^{(2)} m_{2}^{(2)}\left(1 / m_{1} !\right)\left(1 / m_{2} !\right) \sum_{p} \sum_{p^{\prime}} E\left[H_{1, n}\left(Z_{i_{1}}, \ldots, Z_{i_{m_{1}}}\right) H_{2, n}\left(Z_{i_{1}^{\prime}}, \ldots, Z_{i_{m_{2}}^{\prime}}\right) \mid Z_{1}, Z_{2}\right]
$$


Proposition $1 n h^{p / 2} V_{0 n}=n h^{p / 2} U_{0 n}+\delta_{n}^{2} n h^{p / 2} \mu_{n}+\delta_{n} \sqrt{n} h^{p / 2} B_{n}$, where $\mu_{n} \longrightarrow \mu, n h^{p / 2} U_{0 n} \stackrel{d}{\longrightarrow} N\left(0, \omega_{0}^{2}\right)$ and $B_{n} \stackrel{d}{\longrightarrow} 2 N\left(0, \zeta-\delta^{2} \mu^{2}\right)$, with $\delta=\lim _{n \rightarrow \infty} \delta_{n}$ and $\mu, \omega_{0}^{2}$ and $\zeta$ subsequently defined.

Proof: $V_{0 n}=U_{0 n}+W_{0 n}-\theta_{n}$, where $H_{n}\left(Z_{i}, Z_{j}\right)=u_{i} u_{j} f_{i} f_{j} \tilde{K}_{n i j} w_{n i j}, \theta_{n}=E\left[H_{n}\left(Z_{1}, Z_{0}\right)\right], W_{0 n}=$ $(2 / n) \sum_{i} E\left[H_{n}\left(Z_{i}, Z_{0}\right) \mid Z_{i}\right]$ and

$U_{0 n}=\left(\begin{array}{l}n \\ 2\end{array}\right)^{-1} \sum_{i<j} \tilde{H}_{n}\left(Z_{i}, Z_{j}\right)=\left(\begin{array}{l}n \\ 2\end{array}\right)^{-1} \sum_{i<j}\left\{H_{n}\left(Z_{i}, Z_{j}\right)-E\left[H_{n}\left(Z_{i}, Z_{0}\right) \mid Z_{i}\right]-E\left[H_{n}\left(Z_{0}, Z_{j}\right) \mid Z_{j}\right]+\theta_{n}\right\}$.

$$
\begin{aligned}
\theta_{n} & =E\left[u_{i} f_{i} u_{j} f_{j} \tilde{K}_{n i j} w_{n i j}\right]=E\left[\left(U_{i}+\delta_{n} d_{i}\right) f_{i}\left(U_{j}+\delta_{n} d_{j}\right) f_{j} \tilde{K}_{n i j} w_{n i j}\right] \\
& =\delta_{n}^{2} E\left[d_{i} f_{i} d_{j} f_{j} \tilde{K}_{n i j} w_{n i j}\right]=\delta_{n}^{2} E\left[d_{i} f_{i} w_{n i j} E\left(d_{j} f_{j} \tilde{K}_{n i j} \mid X_{i}, C_{i}, C_{j}\right)\right] .
\end{aligned}
$$

Now $E\left[\delta_{n} d_{j} f_{j} \tilde{K}_{n i j} \mid X_{i}, C_{i}, C_{j}\right]=\delta_{n} d\left(X_{i}, C_{j}\right) f\left(X_{i}\right) f_{C_{j}}\left(X_{i}\right)+o\left(\delta_{n}\right)$ uniformly in $X_{i}$ by Lemma 2, as $\delta_{n} d(X, C) f(X) f_{C}(X) \in \mathcal{U}^{p}, \forall C$. Therefore $\theta_{n}=\delta_{n}^{2} E\left[d_{i}^{2} f_{i}^{2} E\left(w_{n i j} \mid C_{i}\right) f_{C_{i}}\left(X_{i}\right)\right]+o\left(\delta_{n}^{2}\right)=\delta_{n}^{2} \mu_{n}$ with $\mu_{n} \longrightarrow \mu=E\left[d^{2}(X, C) f^{2}(X) f_{C}(X)\right]$, as $E\left(w_{n i j} \mid C_{i}\right)=1$.

(ii) Distribution of $W_{0 n}: E\left[E^{2}\left(H_{n}\left(Z_{i}, Z_{0}\right) \mid Z_{i}\right)\right]=E\left[u_{i}^{2} f_{i}^{2} E^{2}\left(u_{0} f_{0} \tilde{K}_{n i 0} w_{n i 0} \mid Z_{i}\right)\right]=\delta_{n}^{2} \zeta_{n}$ with $\zeta_{n} \longrightarrow$ $\zeta=E\left[\sigma_{C}^{2}(X) d^{2}(X, C) f^{4}(X) f_{C}^{2}(X)\right]$, as $\delta_{n} d(X, C) f(X) f_{C}(X) \in \mathcal{U}^{p}, \forall C$.

Now $E\left|E\left[H_{n}\left(Z_{i}, Z_{j}\right) \mid Z_{i}\right]\right|^{\nu}=E\left|u_{i}^{\nu} f_{i}^{\nu} E^{\nu}\left[u_{0} f_{0} \tilde{K}_{n i 0} w_{n i 0} \mid Z_{i}\right]\right|=O(1)=o\left(n^{\nu / 2-1}\right)$ for some $2<\nu \leq 4$, as $E\left|Y^{8}\right|<\infty$. Thus, by Theorem 7.1 of Hoeffding (1948), $\sqrt{n}\left[W_{0 n}-2 \theta_{n}\right] \rightarrow 2 \delta N\left(0, \zeta-\delta^{2} \mu^{2}\right)$.

(iii) Distribution of $U_{0 n}$ : As $E\left[\tilde{H}_{n}\left(Z_{i}, Z_{j}\right) \mid Z_{i}\right]=0$, by Lemma 1 ,

$$
n h^{p / 2} U_{0 n} \stackrel{d}{\longrightarrow} N\left(0, \tau^{2}\right) \quad \text { if } \quad \frac{E\left[\tilde{G}_{n}^{2}\right]+n^{-1} E\left[\tilde{H}_{n}^{4}\right]}{E^{2}\left[\tilde{H}_{n}^{2}\right]}=o(1),
$$

where $\tilde{G}_{n}\left(Z_{i}, Z_{j}\right)=E\left[\tilde{H}_{n}\left(Z_{i}, Z_{0}\right) \tilde{H}_{n}\left(Z_{j}, Z_{0}\right) \mid Z_{i}, Z_{j}\right]$ and $\tau^{2}=2 \lim _{n \rightarrow \infty} h^{p} E\left(\tilde{H}_{n}^{2}\right)$. By definition of $\tilde{H}_{n}\left(Z_{i}, Z_{j}\right)$, the above is equivalent to

$$
\frac{E\left[G_{n}^{2}\right]+n^{-1} E\left[H_{n}^{4}\right]}{E^{2}\left[H_{n}^{2}\right]}=o(1)
$$

where $G_{n}\left(Z_{i}, Z_{j}\right)=E\left[H_{n}\left(Z_{i}, Z_{0}\right) H_{n}\left(Z_{j}, Z_{0}\right) \mid Z_{i}, Z_{j}\right]$, and $\tau^{2}=2 \lim _{n \rightarrow \infty} h^{p} E\left(H_{n}^{2}\right)$.

As $\sigma_{C}^{2}(X) f^{2}(X) f_{C}^{2}(X) \in \mathcal{U}^{p}, \forall C, E\left[H_{n}^{2}\left(Z_{i}, Z_{j}\right)\right]=E\left[\sigma_{C_{i}}^{2}\left(X_{i}\right) \sigma_{C_{j}}^{2}\left(X_{j}\right) f_{i}^{2} f_{j}^{2} \tilde{K}_{n i j}^{2} w_{n i j}^{2}\right]=h^{-p} \omega_{0, n}^{2} / 2$, where $\omega_{0, n}^{2} \longrightarrow \omega_{0}^{2}=2 E\left[\sigma_{C}^{2}(X) \sigma_{C^{\prime}}^{2}(X) w_{C C^{\prime}}^{2} f^{4}(X) \int K_{a_{C}}^{2}(t) d t\right]$, using (2.4).

Moreover, as $E\left(u^{4} \mid X, C\right) f^{4}(X) f_{C}(X) \in \mathcal{U}^{p}, \forall C$,

$$
E\left[H_{n}^{4}\right]=E\left[u_{i}^{4} u_{j}^{4} f_{i}^{4} f_{j}^{4} \tilde{K}_{n i j}^{4} w_{n i j}^{4}\right]=E\left[E\left(u_{i}^{4} \mid X_{i}, C_{i}\right) f_{i}^{4} E\left(u_{j}^{4} \mid X_{j}, C_{j}\right) f_{j}^{4} \tilde{K}_{n i j}^{4} w_{n i j}^{4}\right]=O\left(h^{-3 p}\right) .
$$

As $G_{n}\left(Z_{i}, Z_{j}\right)=u_{i} f_{i} u_{j} f_{j} E\left[\sigma_{C_{0}}^{2}\left(X_{0}\right) f^{2}\left(X_{0}\right) \tilde{K}_{n i 0} \tilde{K}_{n j 0} w_{n i 0} w_{n j 0} \mid Z_{i}, Z_{j}\right]$, we have

$E\left[G_{n}^{2}\right]=\int \sigma_{i}^{2} f_{i}^{2} \sigma_{j}^{2} f_{j}^{2} E\left[\int \sigma_{C_{0}}^{2} \tilde{K}_{n i 0} \tilde{K}_{n j 0} f^{2}\left(X_{0}\right) f_{C_{0}}\left(X_{0}\right) d X_{0} w_{n i 0} w_{n j 0} \mid Z_{i}, Z_{j}\right]^{2} f_{i} f_{j} d X_{i} d X_{j} d \nu\left(C_{i}, C_{j}\right)$ 


$$
\begin{aligned}
& =h^{-p} \int \sigma_{i}^{2} f_{i}^{2} \sigma_{C_{j}}^{2}\left(X_{i}+h t\right) f^{2}\left(X_{i}+h t\right) \\
& \quad E\left[\int \sigma_{C_{0}}^{2}\left(X_{i}-h s\right) K_{a_{C_{0}}}(s) K_{a_{C_{0}}}(s+t) f^{2}\left(X_{i}-h s\right) f_{C_{0}}\left(X_{i}-h s\right) d s w_{n i 0} w_{n j 0} \mid Z_{i}, Z_{j}\right]^{2} \\
& \quad f_{C_{i}}\left(X_{i}\right) f_{C_{j}}\left(X_{i}+h t\right) d X_{i} d t d \nu\left(C_{i}, C_{j}\right) \\
& =h^{-p} E\left[\sigma_{C}^{8}(X) f^{8}(X) f_{C}^{3}(X) \int\left(K_{a_{C}} * K_{a_{C}}\right)^{2}(t) d t\right]+o\left(h^{-p}\right)=O\left(h^{-p}\right),
\end{aligned}
$$

where $\nu\left(C_{i}, C_{j}\right)$ denotes the distribution of $\left(C_{i}, C_{j}\right)$. Thus (A.1) holds as $h \rightarrow 0$ and $n h^{p} \rightarrow \infty$. Q.E.D.

Proposition $2 n h^{p / 2} I_{1,3}=n h^{p / 2} U_{1 n}+\delta_{n} \sqrt{n} h^{p / 2} O_{p}(1)+o_{p}(1)$, where $n h^{p / 2} U_{1 n}$ is asymptotically centered normal with variance $2 E\left[\sigma_{C}^{2}(X) \sigma_{C^{\prime}}^{2}(X) f^{2}(X) f_{C}^{2}(X) \int\left(K * K_{a_{C}}\right)^{2}(t) d t\right]$.

Proof: $I_{1,3}$ is a U-statistic with kernel $H_{n}\left(Z_{i}, Z_{j}, Z_{l}\right)=u_{i} f_{i} u_{l} K_{n j l} \tilde{K}_{n i j} w_{n i j}$. We now compute the corresponding $\xi_{s}, s=1,2,3$.

(i) $\xi_{2}=h^{-p} 2 E\left[\sigma_{C}^{2}(X) \sigma_{C^{\prime}}^{2}(X) f^{2}(X) f_{C}^{2}(X) \int\left(K * K_{a_{C}}\right)^{2}(t) d t\right]+o\left(h^{-p}\right)$. Indeed we have $E\left(H_{n} \mid Z_{i}, Z_{j}\right)=$ $0, E\left(H_{n} \mid Z_{i}, Z_{l}\right)=u_{i} f_{i} u_{l} E\left(K_{n j l} \tilde{K}_{n i j} w_{n i j} \mid Z_{i}, Z_{l}\right)$ and $E\left(H_{n} \mid Z_{j}, Z_{l}\right)=\delta_{n} u_{l} K_{n j l} E\left(d_{i} f_{i} \tilde{K}_{n i j} w_{n i j} \mid Z_{j}\right)$.

Now $E\left[E^{2}\left(H_{n} \mid Z_{i}, Z_{l}\right)\right]=E\left[\sigma_{i}^{2} f_{i}^{2} \sigma_{l}^{2} E^{2}\left(K_{n j l} \tilde{K}_{n i j} w_{n i j} \mid Z_{i}, Z_{l}\right)\right]$

$$
\begin{aligned}
& =h^{-p} E\left[\sigma_{C}^{2}(X) \sigma_{C^{\prime}}^{2}(X) f^{2}(X) f_{C}^{2}(X) \int\left(K * K_{a_{C}}\right)^{2}(t) d t\right]+o\left(h^{-p}\right), \\
E\left[E^{2}\left(H_{n} \mid Z_{j}, Z_{l}\right)\right] & =\delta_{n}^{2} E\left[\sigma_{l}^{2} K_{n j l}^{2} d_{j}^{2} f_{j}^{2} f_{C_{j}}^{2}\left(X_{j}\right)\right]=\delta_{n}^{2} O\left(h^{-p}\right) E\left[u_{l}^{2} \boldsymbol{K}_{n j l} d_{j}^{2} f_{j}^{2} f_{C_{j}}^{2}\left(X_{j}\right)\right]=O\left(\delta_{n}^{2} h^{-p}\right),
\end{aligned}
$$

$$
\begin{aligned}
E & {\left[E\left(H_{n} \mid Z_{i}, Z_{l}\right) E\left(H_{n} \mid Z_{j}, Z_{l}\right)\right] } \\
& =\delta_{n}^{2} E\left[d_{i} f_{i} \sigma_{l}^{2} K_{n j l} E\left(K_{n j l} \tilde{K}_{n i j} w_{n i j} \mid Z_{i}, Z_{l}\right) E\left(d_{i} f_{i} \tilde{K}_{n i j} w_{n i j} \mid Z_{j}\right)\right] \\
& =\delta_{n}^{2} O\left(h^{-p}\right) E\left[\left|d_{i}\right| f_{i} \sigma_{l}^{2} \boldsymbol{K}_{n j l} E\left(\tilde{\boldsymbol{K}}_{n i j} w_{n i j} \mid Z_{i}, Z_{l}\right)\left|E\left(d_{i} f_{i} \tilde{K}_{n i j} w_{n i j} \mid Z_{j}\right)\right|\right]=O\left(\delta_{n}^{2} h^{-p}\right) .
\end{aligned}
$$

(ii) $\xi_{1}=O\left(\delta_{n}^{2}\right)$. Indeed $E\left(H_{n} \mid Z_{i}\right)=E\left(H_{n} \mid Z_{j}\right)=0$ and $E\left(H_{n} \mid Z_{l}\right)=\delta_{n} u_{l} E\left(d_{i} f_{i} K_{n j l} \tilde{K}_{n i j} w_{n i j} \mid Z_{l}\right)$. Then $E\left[E^{2}\left(H_{n} \mid Z_{l}\right)\right]=\delta_{n}^{2} E\left[u_{l}^{2} E^{2}\left(K_{n j l} E\left(d_{i} f_{i} \tilde{K}_{n i j} w_{n i j} \mid Z_{j}, Z_{l}\right) \mid Z_{l}\right)\right]=O\left(\delta_{n}^{2}\right)$.

(iii) $E\left[H_{n}\right]=0$.

(iv) $\xi_{3}=O\left(h^{-2 p}\right)$, as $E\left[H_{n}^{2}\right]=O\left(h^{-2 p}\right) E\left[u_{i}^{2} u_{l}^{2} f_{i}^{2} \boldsymbol{K}_{n j l} \tilde{\boldsymbol{K}}_{n i j} w_{n i j}^{2}\right]=O\left(h^{-2 p}\right)$.

Thus $E\left[n h^{p / 2}\left(I_{1,3}-U_{1 n}\right)\right]^{2}=\delta_{n}^{2} n h^{p} O(1)$, where $U_{1 n}=\left(1 / n^{(3)}\right) \sum_{a}\left[H_{n}\left(Z_{i}, Z_{j}, Z_{l}\right)-E\left(H_{n} \mid Z_{l}\right)\right]$. Similarly, $E\left[H_{n, 2}^{4}\right]=O\left(h^{-3 p}\right)$ and $E\left[G_{n}^{2}\right]=O\left(h^{-p}\right)$. The result then follows from Lemma $1 . \quad$ Q.E.D.

Proposition $3 n h^{p / 2} I_{1,5}$ is asymptotically centered normal with variance $2 E\left[\sigma_{C}^{2}(X) \sigma_{C^{\prime}}^{2}(X) \int g^{4}(X, t) d t\right]$, where $g^{2}(x, t)=\sum_{c} p_{c} f_{c}^{2}(x)\left(K * K * K_{a_{c}}\right)^{2}(t)$.

Proof: $I_{1,5}$ is a U-statistic with kernel $H_{n}\left(Z_{i}, Z_{j}, Z_{k}, Z_{l}\right)=u_{k} u_{l} K_{n i k} K_{n j l} \tilde{K}_{n i j} w_{n i j}$. 
(i) $\xi_{3}=O\left(h^{-2 p}\right)$. Indeed we have $E\left(H_{n} \mid Z_{i}, Z_{j}, Z_{k}\right)=E\left(H_{n} \mid Z_{i}, Z_{j}, Z_{l}\right)=0$, and

$$
\begin{aligned}
& E\left(H_{n} \mid Z_{i}, Z_{k}, Z_{l}\right)=u_{k} u_{l} K_{n i k} E\left(K_{n j l} \tilde{K}_{n i j} w_{n i j} \mid Z_{i}, Z_{l}\right), \\
& E\left(H_{n} \mid Z_{j}, Z_{k}, Z_{l}\right)=u_{k} u_{l} K_{n j l} E\left(K_{n i k} \tilde{K}_{n i j} w_{n i j} \mid Z_{j}, Z_{k}\right) .
\end{aligned}
$$

Then $E\left[E^{2}\left(H_{n} \mid Z_{i}, Z_{k}, Z_{l}\right)\right]=E\left[u_{k}^{2} u_{l}^{2} K_{n i k}^{2} E\left(K_{n j l} \tilde{K}_{n i j} w_{n i j} \mid Z_{i}, Z_{l}\right) E\left(K_{n j^{\prime} l} \tilde{K}_{n i j^{\prime}} w_{n i j^{\prime}} \mid Z_{i}, Z_{l}\right)\right]$

$$
\begin{aligned}
& =O\left(h^{-2 p}\right) E\left[u_{k}^{2} u_{l}^{2} \boldsymbol{K}_{n i k} E\left(\boldsymbol{K}_{n j l} \tilde{\boldsymbol{K}}_{n i j} w_{n i j} \mid Z_{i}, Z_{l}\right) E\left(\tilde{\boldsymbol{K}}_{n i j^{\prime}} w_{n i j^{\prime}} \mid Z_{i}, Z_{l}\right)\right] \\
& =O\left(h^{-2 p}\right) E\left[u_{k}^{2} u_{l}^{2} \boldsymbol{K}_{n i k} \boldsymbol{K}_{n j l} \tilde{\boldsymbol{K}}_{n i j} w_{n i j} f_{C i}^{2}\left(X_{i}\right)\right]=O\left(h^{-2 p}\right),
\end{aligned}
$$

Similarly $E\left[E^{2}\left(H_{n} \mid Z_{j}, Z_{k}, Z_{l}\right)\right]$ and $E\left[E\left(H_{n} \mid Z_{i}, Z_{k}, Z_{l}\right) E\left(H_{n} \mid Z_{j}, Z_{k}, Z_{l}\right)\right]$ are $O\left(h^{-2 p}\right)$.

(ii) $\xi_{2}=h^{-p} 2 E\left[\sigma_{C}^{2}(X) \sigma_{C^{\prime}}^{2} \int g^{4}(X, t) d t\right]+o_{p}\left(h^{-p}\right)$. Indeed we have $E\left(H_{n} \mid Z_{i}, Z_{j}\right)=E\left(H_{n} \mid Z_{i}, Z_{k}\right)=$ $E\left(H_{n} \mid Z_{i}, Z_{l}\right)=E\left(H_{n} \mid Z_{j}, Z_{k}\right)=E\left(H_{n} \mid Z_{j}, Z_{l}\right)=0$ and $E\left(H_{n} \mid Z_{k}, Z_{l}\right)=u_{k} u_{l} E\left(K_{n i k} K_{n j l} \tilde{K}_{n i j} w_{n i j} \mid Z_{k}, Z_{l}\right)$, so that $E\left[E^{2}\left(H_{n} \mid Z_{k}, Z_{l}\right)\right]=E\left[\sigma_{k}^{2} \sigma_{l}^{2} E^{2}\left(K_{n i k} K_{n j l} \tilde{K}_{n i j} w_{n i j} \mid Z_{k}, Z_{l}\right)\right]$. Now

$$
\begin{gathered}
E\left(K_{n i k} K_{n j l} \tilde{K}_{n i j} w_{n i j} \mid Z_{k}, Z_{l}\right)=E\left[w_{n i j} \int K_{n i k} K_{n j l} \tilde{K}_{n i j} f_{C_{i}}\left(X_{i}\right) f_{C_{j}}\left(X_{j}\right) d X_{i} d X_{j} \mid Z_{k}, Z_{l}\right] \\
=E\left[w_{n i j} f_{C_{i}}\left(X_{k}\right) f_{C_{j}}\left(X_{l}\right) \int K(u) K(v) h^{-p} K_{a_{C_{i}}}\left(u-v+h^{-1}\left(X_{k}-X_{l}\right)\right) d u d v \mid Z_{k}, Z_{l}\right]
\end{gathered}
$$

uniformly in $\left(X_{i}, X_{j}\right)$ as $f_{C}(\cdot) \in \mathcal{U}^{p}, \forall C$. Therefore

$$
\begin{aligned}
& E {\left[E^{2}\left(H_{n} \mid Z_{k}, Z_{l}\right)\right] } \\
&=\int \sigma_{k}^{2} \sigma_{l}^{2}\left[\int w_{n i j} f_{C_{i}}\left(X_{k}\right) f_{C_{j}}\left(X_{l}\right) \int K(u) K(v) h^{-p} K_{a_{C_{i}}}\left(u-v+h^{-1}\left(X_{k}-X_{l}\right)\right) d u d v d \nu\left(C_{i}, C_{j}\right)\right]^{2} \\
& f_{C_{k}}\left(X_{k}\right) f_{C_{l}}\left(X_{l}\right) d X_{k} d X_{l} d \nu\left(C_{k}, C_{l}\right)
\end{aligned}
$$

where $g^{2}(x, s)=\sum_{c} p_{c} f_{c}^{2}(x)\left(K * K * K_{a_{c}}\right)^{2}(s)$.

(iii) $\xi_{1}=0$, as $E\left(H_{n} \mid Z_{i}\right)=E\left(H_{n} \mid Z_{j}\right)=E\left(H_{n} \mid Z_{k}\right)=E\left(H_{n} \mid Z_{l}\right)=0$.

(iv) $E\left[H_{n}\right]=0$.

(v) $\xi_{4}=O\left(h^{-3 p}\right)$, as $E\left[H_{n}^{2}\right]=E\left[u_{k}^{2} u_{l}^{2} K_{n i k}^{2} K_{n j l}^{2} \tilde{K}_{n i j}^{2} w_{n i j}^{2}\right]=O\left(h^{-3 p}\right) E\left[u_{k}^{2} u_{l}^{2} \boldsymbol{K}_{n i k} \boldsymbol{K}_{n j l} \tilde{\boldsymbol{K}}_{n i j} w_{n i j}^{2}\right]=$ $O\left(h^{-3 p}\right)$.

Similarly $E\left[H_{n, 2}^{4}\right]=O\left(h^{-3 p}\right)$ and $E\left[G_{n}^{2}\right]=O\left(h^{-p}\right)$. The result follows from Lemma 1.

Q.E.D.

Proposition $4 n h^{p / 2}\left(U_{0 n}-2 U_{1 n}+I_{1,5}\right) \stackrel{d}{\longrightarrow} N\left(0, \omega^{2}\right)$. 
Proof: To show this, we apply the Cramer-Wold device. We compute the covariances between $U_{0 n}, U_{1 n}$ and $I_{1,5}$.

Covariance Between $U_{0 n}$ AND $U_{1 n}: \xi_{2,2}$ is determined by

$$
\begin{gathered}
E\left[u_{i} f_{i} u_{l} f_{l} \tilde{K}_{n i l} w_{n i l} E\left(u_{i} f_{i} u_{l} K_{n j l} \tilde{K}_{n i j} w_{n i j} \mid Z_{i}, Z_{l}\right)\right]=E\left[\sigma_{i}^{2} f_{i}^{2} \sigma_{l}^{2} f_{l} \tilde{K}_{n i l} w_{n i l} E\left(K_{n j l} \tilde{K}_{n i j} w_{n i j} \mid Z_{i}, Z_{l}\right)\right] \\
=h^{-p} E\left[\sigma_{C}^{2}(X) \sigma_{C^{\prime}}^{2}(X) w_{C C^{\prime}} f^{3}(X) f_{C}(X) \int K_{a_{C}}(t)\left(K * K_{a_{C}}\right)(t) d t\right]+o\left(h^{-p}\right) .
\end{gathered}
$$

Covariance Between $U_{0 n}$ And $I_{1,5}: \xi_{2,2}$ is determined by

$$
\begin{aligned}
E & {\left[u_{k} f_{k} u_{l} f_{l} \tilde{K}_{n k l} w_{n k l} E\left(u_{k} u_{l} K_{n i k} K_{n j l} \tilde{K}_{n i j} w_{n i j} \mid Z_{k}, Z_{l}\right)\right] } \\
& =E\left[\sigma_{k}^{2} f_{k} \sigma_{l}^{2} f_{l} \tilde{K}_{n k l} w_{n k l} E\left(K_{n i k} K_{n j l} \tilde{K}_{n i j} w_{n i j} \mid Z_{k}, Z_{l}\right)\right] \\
& =h^{-p} E\left[\sigma_{C}^{2}(X) \sigma_{C^{\prime}}^{2}(X) w_{C C^{\prime}} f^{2}(X) f_{C^{\prime \prime}}^{2}(X) \int K_{a_{C}}(t)\left(K * K * K_{a_{C^{\prime \prime}}}\right)(t) d t\right]+o\left(h^{-p}\right) .
\end{aligned}
$$

Covariance Between $U_{1 n}$ And $I_{1,5}: \xi_{3,3}=O\left(h^{-p}\right)$. and $\xi_{2,2}$ is determined by

$$
\begin{aligned}
E & {\left[E\left(u_{k} f_{k} u_{l} K_{n j l} \tilde{K}_{n k j} w_{n k j} \mid Z_{k}, Z_{l}\right) E\left(u_{k} u_{l} K_{n i k} K_{n j^{\prime} l} \tilde{K}_{n i j^{\prime}} w_{n i j^{\prime}} \mid Z_{k}, Z_{l}\right)\right] } \\
& =E\left[\sigma_{k}^{2} f_{k} \sigma_{l}^{2} E\left(K_{n j l} \tilde{K}_{n k j} w_{n k j} \mid Z_{k}, Z_{l}\right) E\left(K_{n i k} K_{n j^{\prime} l} \tilde{K}_{n i j^{\prime}} w_{n i j^{\prime}} \mid Z_{k}, Z_{l}\right)\right] \\
& =h^{-p} E\left[\sigma_{C}^{2}(X) \sigma_{C^{\prime}}^{2}(X) f(X) f_{C}(X) f_{C^{\prime \prime}}^{2}(X) \int\left(K * K_{a_{C}}\right)(t)\left(K * K * K_{a_{C}^{\prime \prime}}\right)(t) d t\right]+o\left(h^{-p}\right) .
\end{aligned}
$$

Hence $n h^{p / 2}\left[U_{0 n}-2 U_{1 n}+I_{1,5}\right] \stackrel{d}{\longrightarrow} N\left(0, \omega^{2}\right)$, where

$$
\begin{aligned}
\omega^{2}= & 2 E\left[\sigma_{C}^{2}(X) \sigma_{C^{\prime}}^{2}(X) w_{C C^{\prime}}^{2} f^{4}(X) \int K_{a_{C}}^{2}(t) d t\right]+2 E\left[\sigma_{C}^{2}(X) \sigma_{C^{\prime}}^{2}(X) \int g^{4}(X, t) d t\right] \\
& +8 E\left[\sigma_{C}^{2}(X) \sigma_{C^{\prime}}^{2}(X) f^{2}(X) f_{C}^{2}(X) \int\left(K * K_{a_{C}}\right)^{2}(t) d t\right] \\
& -8 E\left[\sigma_{C}^{2}(X) \sigma_{C^{\prime}}^{2}(X) w_{C C^{\prime}} f^{3}(X) f_{C}(X) \int K_{a_{C}}(t)\left(K * K_{a_{C}}\right)(t) d t\right] \\
& +4 E\left[\sigma_{C}^{2}(X) \sigma_{C^{\prime}}^{2}(X) w_{C C^{\prime}} f^{2}(X) f_{C^{\prime \prime}}^{2}(X) \int K_{a_{C}}(t)\left(K * K * K_{a_{C}^{\prime \prime}}\right)(t) d t\right] \\
& -8 E\left[\sigma_{C}^{2}(X) \sigma_{C^{\prime}}^{2}(X) f(X) f_{C}(X) f_{C^{\prime \prime}}^{2}(X) \int\left(K * K_{a_{C}}\right)(t)\left(K * K * K_{a_{C}^{\prime \prime}}\right)(t) d t\right] \\
= & 2 E\left[\sigma_{C}^{2}(X) \sigma_{C^{\prime}}^{2} f^{4}(X) E_{C C^{\prime}}(X)\right],
\end{aligned}
$$

with $E_{C C^{\prime}}(X)=\int\left[K_{a_{C}}(t) w_{C C^{\prime}}-2\left(K * K_{a_{C}}\right)(t) \frac{f_{C}(X)}{f(X)}+\frac{g^{2}(X, t)}{f^{2}(X)}\right]^{2} d t$.

Q.E.D.

Proposition $5 n h^{p / 2} I_{1,6}=\delta_{n}^{2} n h^{p / 2} o_{p}(1)+o_{p}(1)$.

Proposition $6 n h^{p / 2} I_{2,1}=\delta_{n} \sqrt{n} h^{p / 2} o_{p}(1)+\delta_{n} n h^{p / 2} h^{(m+q)} O_{p}(1)+o_{p}(1)$.

Proposition $7 n h^{p / 2} I_{2,3}=o_{p}(1)$. 
Proposition $8 n h^{p / 2} I_{3}=n h^{p / 2} O_{p}\left(h^{2(m+q)}\right)+o_{p}(1)$.

Proofs of Propositions 5-8: They follow the same lines as the ones of Proposition 4 to 7 by Lavergne and Vuong (2000), provided that $K_{n i j}$ is replaced by $\tilde{K}_{n i j}, L(\cdot)$ by $K(\cdot)$ and $g$ by $h$.

Proposition $9 n h^{p / 2} I_{1,1}=\delta_{n}^{2} n h^{p / 2} o_{p}(1)+\delta_{n} \sqrt{n} h^{p / 2} o_{p}(1)+o_{p}(1)$.

Proof: Let $\Delta f_{i}^{j} \equiv\left(\widehat{f}_{i}^{j}-f_{i}\right)$. We have $I_{1,1}=\left(1 / n^{(2)}\right) \sum_{a} u_{i} \Delta f_{i}^{j} u_{j} f_{j} \tilde{K}_{n i j} w_{n i j}$ so that

$$
E\left(I_{1,1}^{2}\right)=\left(\frac{1}{n^{(2)}}\right)^{2}\left[\sum_{a} u_{i} \Delta f_{i}^{j} u_{j} f_{j} \tilde{K}_{n i j} w_{n i j}\right]\left[\sum_{a} u_{i^{\prime}} \Delta f_{i^{\prime}}^{j^{\prime}} u_{j^{\prime}} f_{j^{\prime}} K_{n i^{\prime} j^{\prime}} w_{n i^{\prime} j^{\prime}}\right],
$$

where the sums are taken over all arrangements of different indices In what follows, $\tilde{K}_{n i j} \equiv \tilde{K}_{n i j} w_{n i j}$.

(i) All indices are different: $n^{(4)}$ terms.

$$
\begin{aligned}
E\left[u_{i} \Delta f_{i}^{j} u_{j} f_{j} \tilde{K}_{n i j} u_{i^{\prime}} \Delta f_{i^{\prime}}^{j^{\prime}} u_{j^{\prime}} f_{j^{\prime}} \tilde{K}_{n i^{\prime} j^{\prime}}\right] & =\delta_{n}^{4} E\left[\Delta f_{i}^{j} f_{j} \Delta f_{i^{\prime}}^{j^{\prime}} f_{j^{\prime}} d_{i} d_{j} d_{i^{\prime}} d_{j^{\prime}} \tilde{K}_{n i j} \tilde{K}_{n i^{\prime} j^{\prime}}\right] \\
& =\delta_{n}^{4} E\left[f_{j} f_{j^{\prime}} d_{i} d_{j} d_{i^{\prime}} d_{j^{\prime}} \tilde{K}_{n i j} \tilde{K}_{n i^{\prime} j^{\prime}} E\left(\Delta f_{i}^{j} \Delta f_{i^{\prime}}^{j^{\prime}} \mid Z_{i}, Z_{j}, Z_{i^{\prime}}, Z_{j^{\prime}}\right)\right] \\
& =\delta_{n}^{4} \lambda_{n} E\left|f_{j} f_{j^{\prime}} d_{i} d_{j} d_{i^{\prime}} d_{j^{\prime}} \tilde{K}_{n i j} \tilde{K}_{n i^{\prime} j^{\prime}}\right|=O\left(\delta_{n}^{4} \lambda_{n}\right),
\end{aligned}
$$

where $\lambda_{n}=E\left[\Delta^{2} f_{i}^{j} \mid Z_{i}, Z_{j}, Z_{i^{\prime}}, Z_{j^{\prime}}\right]$.

(ii) One index is common to $\{i, j\}$ and $\left\{i^{\prime}, j^{\prime}\right\}$ : $4 n^{(3)}$ terms.

$$
\begin{array}{ll}
\left(i^{\prime}=i\right) & E\left[u_{i}^{2} \Delta f_{i}^{j} u_{j} f_{j} \tilde{K}_{n i j} \Delta f_{i}^{j^{\prime}} u_{j^{\prime}} f_{j^{\prime}} \tilde{K}_{n i j^{\prime}}\right]=\delta_{n}^{2} \lambda_{n} E\left|f_{j} f_{j^{\prime}} u_{i}^{2} d_{j} d_{j^{\prime}} \tilde{K}_{n i j} \tilde{K}_{n i j^{\prime}}\right|=O\left(\delta_{n}^{2} \lambda_{n}\right), \\
\left(j^{\prime}=j\right) & E\left[u_{i} \Delta f_{i}^{j} u_{j}^{2} f_{j}^{2} \tilde{K}_{n i j} u_{i^{\prime}} \Delta f_{i^{\prime}}^{j} \tilde{K}_{n i^{\prime} j}\right]=\delta_{n}^{2} \lambda_{n} E\left|f_{j}^{2} d_{i} u_{j}^{2} d_{i^{\prime}} \tilde{K}_{n i j} \tilde{K}_{n i^{\prime} j}\right|=O\left(\delta_{n}^{2} \lambda_{n}\right), \\
\left(i^{\prime}=j\right) & E\left[u_{i} \Delta f_{i}^{j} u_{j}^{2} f_{j} \tilde{K}_{n i j} \Delta f_{j}^{j^{\prime}} u_{j^{\prime}} f_{j^{\prime}} \tilde{K}_{n j j^{\prime}}\right]=\delta_{n}^{2} \lambda_{n} E\left|f_{j} f_{j^{\prime}} d_{i} u_{j}^{2} d_{j^{\prime}} \tilde{K}_{n i j} \tilde{K}_{n j j^{\prime}}\right|=O\left(\delta_{n}^{2} \lambda_{n}\right) .
\end{array}
$$

The case $j^{\prime}=i$ is similar to $i^{\prime}=j$.

(iii) Two indices in common to $\{i, j\}$ and $\left\{i^{\prime}, j^{\prime}\right\}$ : $2 n^{(2)}$ terms. We have $E\left[u_{i}^{2} u_{j}^{2}\left(\Delta f_{i}^{j}\right)^{2} f_{j}^{2} \tilde{K}_{n i j}^{2}\right]=$ $O\left(\lambda_{n} / h^{p}\right)$ and $E\left[u_{i}^{2} u_{j}^{2} \Delta f_{i}^{j} \Delta f_{j}^{i} f_{i} f_{j} \tilde{K}_{n i j}^{2}\right]=O\left(\lambda_{n} / h^{p}\right)$.

Therefore, $E\left(n h^{p / 2} I_{1,1}\right)^{2}=\delta_{n}^{4} n^{2} h^{p} O\left(\lambda_{n}\right)+\delta_{n}^{2} n h^{p} O\left(\lambda_{n}\right)+O\left(\lambda_{n}\right)$. The proposition then follows from $\lambda_{n}=o(1)$ uniformly, see Lemma 3.

Q.E.D.

Proposition $10 n h^{p / 2} I_{1,2}=\delta_{n}^{2} n h^{p / 2} o_{p}(1)+\delta_{n} \sqrt{n} h^{p / 2} o_{p}(1)+o_{p}(1)$.

Proposition $11 n h^{p / 2} I_{1,4}=\delta_{n}^{2} n h^{p / 2} o_{p}(1)+\delta_{n} \sqrt{n} h^{p / 2} o_{p}(1)+o_{p}(1)$.

Proposition $12 n h^{p / 2} I_{2,2}=\delta_{n}^{2} n h^{p / 2} o_{p}(1)+\delta_{n} \sqrt{n} h^{p / 2} o_{p}(1)+o_{p}(1)$.

Proofs of Propositions 10-12: They are very similar to Proposition 11's proof and are not reported. The two last proofs use the second part of Lemma 3. 


\section{A.2 Proof of Theorem 2}

The consistency of both forms of $\omega_{n}^{2}$ can be proven using similar arguments as in the proof of Theorem 1 and as in Part (i) of Theorem 1 of Lavergne and Vuong (1996) for the treatment of the trimming parameter. In particular, $\left(b_{n} \sqrt{n} h_{n}^{p}\right)^{-1}=o(1)$ and $b_{n}^{-1} h^{m+q}=o(1)$ ensures that $b_{n}^{-1} \sup _{x \in \mathbb{R}} \mid f_{n}(x)-$ $f(x) \mid=o_{p}(1)$. Hence, $f_{n}(\cdot)$ can be replaced by $f(\cdot)$ in $E_{n i j}$ without changing the probability limit of $\omega_{n}^{2}$.

\section{A.3 Proof of Theorem 3}

We use similar arguments to that in Mammen (1992), who deals with testing equality of density functions. However, the technical analysis is long and tedious, so that we only provide a brief sketch of the proof in the special case where $a_{c}=1, c=1, \ldots, \bar{C}$.

Let us explicit the dependence of $V_{n}$ on the bandwidth. Under the null hypothesis, we shall show that $n \widehat{h}^{p / 2} V_{n}(\widehat{h})-n h^{p / 2} V_{n}(h)=o_{p}(1)$. For this equality to hold, we need to show tightness of the process $n(\nu h)^{p / 2} V_{n}(\nu h)$ for $\nu \in\left[B_{1}, B_{2}\right]$, with $0<B_{1}<1<B_{2}<\infty$. Now $n(\nu h)^{p / 2} V_{n}(\nu h)$ converges in distribution to the same limit for any $\nu$. Hence it is sufficient to show that for $\nu_{1}, \nu_{2} \in\left[B_{1}, B_{2}\right]$,

$$
E\left[n\left(\nu_{1} h\right)^{p / 2} V_{n}\left(\nu_{1} h\right)-n\left(\nu_{2} h\right)^{p / 2} V_{n}\left(\nu_{2} h\right)\right]^{2}=O\left[\left(\nu_{1}-\nu_{2}\right)^{2}\right],
$$

see Billingsley (1968). Now because $\left[\sum_{i=1}^{11} \lambda_{i}\right]^{2} \leq 11 \sum_{i=1}^{n} \lambda_{i}^{2}$, it is sufficient to show an analogous result for each of the eleven terms of Propositions 1 to 3 and 5 to 12. For instance, we can show that

$$
E\left[n\left(\nu_{1} h\right)^{p / 2} V_{0 n}\left(\nu_{1} h\right)-n\left(\nu_{2} h\right)^{p / 2} V_{0 n}\left(\nu_{2} h\right)\right]^{2}=O(1) \int\left[\nu_{1}^{-p / 2} K\left(x / \nu_{1}\right)-\nu_{2}^{-p / 2} K\left(x / \nu_{2}\right)\right] d x
$$

is $O\left[\left(\nu_{1}-\nu_{2}\right)^{2}\right]$ by a Taylor expansion of $\nu_{2}^{-p / 2} K\left[x / \nu_{2}\right]$ around $\nu_{1}$.

Under the alternative hypothesis, it is sufficient to show that for $\nu_{1}, \nu_{2} \in\left[B_{1}, B_{2}\right]$,

$$
E\left[V_{n}\left(\nu_{1} h\right)-V_{n}\left(\nu_{2} h\right)\right]^{2}=O\left[\left(\nu_{1}-\nu_{2}\right)^{2}\right]
$$

An analogous result for $\omega_{n}^{2}$ then implies the desired result.

\section{A.4 Proof of Theorem 4}

The proof of Theorem 4 is analogous to the proof of Theorem 1. To deal with $V_{0 n}, I_{1,3}$ and $I_{1,5}$, we use a straightforward generalization of Lemma 1, which accounts for the fact that observations may not be identically distributed across subsamples, although they are independent. This result is not formally stated, but one can easily check that it holds by examining the proofs of Lemma B.4 of Fan and Li (1996) and of Theorem 1 of Hall (1984): the latter proof relies on a martingale central limit theorem that still applies, see Hall and Heyde (1980, Chapter 3). 


\section{A.5 Panel data}

Under our assumptions, $u_{i t} \equiv Y_{i t}-r\left(X_{i t}\right)$ is independent of $\mathcal{Z}_{i t-1}=\left\{Y_{i 1}, \ldots, Y_{i t-1}, X_{i 1}, \ldots, X_{i t-1}\right\}$ conditionally on $\left\{X_{i t}, l\right\}$ and $E\left[u_{i t} \mid \mathcal{Z}_{i t-1}, X_{i t}, l\right]=R_{t}\left(X_{i t}\right)-r\left(X_{i t}\right)$ is zero under $H_{0}$. Also by assumption $E\left[u_{i t} u_{j t^{\prime}} \mid \mathcal{Z}_{i t-1}, X_{i t}, \mathcal{Z}_{j t^{\prime}-1}, X_{j t^{\prime}}, l\right]=0, \forall i, j, t \neq t^{\prime}$. Then $U_{0, n}, U_{1, n}$ and $I_{1,5}$ are degenerate U-statistics under $H_{0}$ and a generalization of Lemma 1 can be applied. The remaining terms are dealt with as in the proof of Theorem 1, using Lemma 4 for $I_{1,1}, I_{1,2}, I_{1,4}$ and $I_{2,2}$.

\section{Appendix B: Technical lemmas}

Lemma 2 For $l(\cdot) \in \mathcal{U}^{p}, \sup _{x \in \mathbb{R}^{p}}\left|\int l(X) \frac{1}{h^{p}} K\left(\frac{x-X}{h}\right) d X-l(x)\right| \rightarrow 0$ when $h \rightarrow 0$.

Proof: This result comes from the well-known Bochner lemma.

Q.E.D.

Lemma $3 E\left[\left(\hat{f}_{i}^{j}-f_{i}\right)^{2} \mid Z_{i}, Z_{j}, Z_{i^{\prime}}, Z_{j^{\prime}}\right]=o(1)$ and $E\left[\left(\hat{f}_{i}^{j, l}-f_{i}\right)^{2} \mid Z_{i}, Z_{j}, Z_{l}, Z_{i^{\prime}}, Z_{j^{\prime}}, Z_{l^{\prime}}\right]=o(1)$ uniformly in their arguments, if $f_{c}(X) \in \mathcal{U}^{p} \quad \forall c$ and $n h^{p} \rightarrow \infty$.

Proof: The first expectation is

$$
\left.E\left[\left(\widehat{f}_{i}^{j}-E\left(\widehat{f}_{i}^{j} \mid Z_{i}, Z_{j}, Z_{i^{\prime}}, Z_{j^{\prime}}\right)\right)^{2} \mid Z_{i}, Z_{j}, Z_{i^{\prime}}, Z_{j^{\prime}}\right]+\left[E\left(\widehat{f}_{i}^{j} \mid Z_{i}, Z_{j}, Z_{i^{\prime}}, Z_{j^{\prime}}\right)\right)-f_{i}\right]^{2} .
$$

Because $\widehat{f}_{i}^{j}-E\left(\widehat{f}_{i}^{j} \mid Z_{i}, Z_{j}, Z_{i^{\prime}}, Z_{j^{\prime}}\right)=(n-2)^{-1} \sum_{k \notin\left\{i, j, i^{\prime}, j^{\prime}\right\}}\left(K_{n i k}-E\left(K_{n i k} \mid Z_{i}\right)\right)$, whose summands are independent with zero mean conditional on $Z_{i}$,

$$
\begin{aligned}
& E\left[\left(\widehat{f}_{i}^{j}-E\left(\widehat{f}_{i}^{j} \mid Z_{i}, Z_{j}, Z_{i^{\prime}}, Z_{j^{\prime}}\right)\right)^{2} \mid Z_{i}, Z_{j}, Z_{i^{\prime}}, Z_{j^{\prime}}\right] \leq(n-2)^{-2} \sum_{k \notin\left\{i, j, i^{\prime}, j^{\prime}\right\}} E\left[\left(K_{n i k}-E\left(K_{n i k} \mid Z_{i}\right)\right)^{2} \mid Z_{i}\right] \\
& \leq(n-2)^{-2} \sum_{k \notin\left\{i, j, i^{\prime}, j^{\prime}\right\}} E\left[K_{n i k}^{2} \mid Z_{i}\right]=O\left(n h^{p}\right)^{-1} . \\
& \text { Now } \quad \begin{aligned}
{\left[E\left(\widehat{f}_{i}^{j} \mid Z_{i}, Z_{j}, Z_{i^{\prime}}, Z_{j^{\prime}}\right)-f_{i}\right]^{2} } & = \\
& {\left[\frac{1}{n-2}\left(K_{n i i^{\prime}}+K_{n i j^{\prime}}-f_{i}\right)+\frac{n-4}{n-2} E\left(K_{n i k}-f_{i} \mid Z_{i}\right)\right]^{2} } \\
\leq & {\left[O\left(n^{-1} h^{-p}\right)+O\left(n^{-1}\right)+o(1)\right]^{2}=o(1), }
\end{aligned}
\end{aligned}
$$

where we use $f_{i}=\sum_{c} p_{c} f_{c}\left(X_{i}\right)$. The proof for the second part is similar and is not reported. Q.E.D.

Lemma 4 The result of Lemma 3 holds for panel data.

Proof: The proof follows Lemma 3's proof, with the difference that

$$
\begin{aligned}
& E\left[\left(\widehat{f}_{i}^{j}-E\left(\widehat{f}_{i}^{j} \mid Z_{i}, Z_{j}, Z_{i^{\prime}}, Z_{j^{\prime}}\right)\right)^{2} \mid Z_{i}, Z_{j}, Z_{i^{\prime}}, Z_{j^{\prime}}\right] \\
& \quad \leq(n-2)^{-2} \sum_{k, k^{\prime} \notin\left\{i, j, i^{\prime}, j^{\prime}\right\}} E\left[\left(K_{n i k}-E\left(K_{n i k} \mid Z_{i}\right)\right)\left(K_{n i k^{\prime}}-E\left(K_{n i k^{\prime}} \mid Z_{i}\right)\right) \mid Z_{i}\right] .
\end{aligned}
$$


By conditioning upon $\left(Z_{i}, Z_{k}\right)$, all terms such that $k$ and $k^{\prime}$ correspond to different individuals vanishes. At most $n T$ terms correspond to same individuals and all are $O\left(h^{p}\right)^{-1}$ by the Cauchy-Schwartz inequality. Thus the right-hand side is $O\left(n h^{p}\right)^{-1}$ and Lemma 4 follows.

Q.E.D.

\section{Appendix C}

We thereafter give the integrals that enter $\omega^{2}$ when $K(\cdot)$ is uniform on $[-1 / 2,1 / 2]$.

$$
\begin{aligned}
& \int K_{a}^{2}(s) d s \\
& \int K_{a}(s)\left(K * K_{a}\right)(s) d s \\
& =\frac{1}{a} \\
& =\left\{\begin{array}{cc}
1 & \text { if } a<1 / 2 \\
\frac{-1+4 a}{4 a^{2}} & \text { if } a \geq 1 / 2
\end{array}\right. \\
& \int\left(K * K_{a}\right)^{2}(s) d s \\
& =\int K_{a}(s)\left(K * K * K_{a}\right)(s) d s \\
& =\left\{\begin{array}{cl}
1-\frac{a}{3} & \text { if } a<1 \\
\frac{-1+3 a}{3 a^{2}} & \text { if } a \geq 1
\end{array}\right. \\
& \int\left(K * K_{a}\right)(s)\left(K * K * K_{a}\right)(s) d s=\left\{\begin{array}{cc}
\frac{3}{4}-\frac{a^{2}}{6} & \text { if } a<1 / 2 \\
\frac{3-24 a+216 a^{2}-96 a^{3}+16 a^{4}}{192 a^{2}} & \text { if } 1 / 2 \leq a \leq 3 / 2 \\
\frac{-13+32 a^{2}}{32 a^{2}} & \text { if } a>3 / 2
\end{array}\right. \\
& \int\left(K * K * K_{a}\right)^{2}(s) d s \\
& =\left\{\begin{array}{cc}
\frac{40-10 a^{2}+3 a^{3}}{60} & \text { if } a<1 \\
\frac{4-20 a+80 a^{2}-40 a^{3}+10 a^{4}-a^{5}}{60 a^{2}} & \text { if } 1 \leq a \leq 2 \\
\frac{-7+15 a^{2}}{15 a^{2}} & \text { if } a>2 .
\end{array}\right.
\end{aligned}
$$




\section{REFERENCES}

Aït-Sahalia, Y., P. Bickel and T. M. Stoker, 1994, Goodness-of-fit tests for regression using kernel methods, University of Chicago.

Alvarez, J. and M. Arellano, 1998, The time series and cross-section asymptotics of dynamic panel data estimators, CEMFI.

Baltagi, B.H., J. Hidalgo and Q. Li, 1996, A nonparametric test for poolability using panel data Journal of Econometrics , 75, 345-367.

Bierens, H.J., 1987, Kernel estimators of regression functions, in: T. Bewley, ed., Advances in Econometrics (Cambridge University Press, Cambridge) 99-144.

Billingsley, P., 1968, Convergence of Probability Measures (John Wiley \& Sons, New-York).

Chamberlain, G., 1984, Panel data, in: Z. Griliches and M.D. Intriligator, eds., Handbook of Econometrics, Vol. 2 (North-Holland: Amsterdam) Chap. 5, 285-330.

Chow, G.C., 1960, Tests of equality between sets of coefficients in two linear regressions, Econometrica 28(3), $591-605$.

Delgado, M.A., 1993, Testing the equality of nonparametric regression curves, Statistics and Probability Letters $17,199-204$.

Delgado, M.A., M.A. Dominguez and P. Lavergne, 1998, Asymptotic and bootstrap specification tests of nonlinear in variables econometric models, Universidad Carlos III de Madrid.

Delgado, M.A. and J. Mora, 1995, Nonparametric and semiparametric estimation with discrete regressors, Econometrica 63(6), 1477-1484.

Fan, J. and S.-K. Lin, 1998, Test of significance when data are curves, Journal of the American Statistical Association 93 (443), 1007-1021.

Fan, Y. and Q. Li, 1996, Consistent model specification tests: omitted variables and semiparametric functional forms, Econometrica 64 (4), 865-890.

Gozalo, P.L., 1995, Nonparametric specification testing with $\sqrt{n}$ - local power and bootstrap critical values, Brown University.

Guerre, E. and P. Lavergne, 1999, Minimax rates for nonparametric specification testing in regression models, LSTA, Université Paris 6.

Hall, P., 1984, Central limit theorem for integrated square error of multivariate nonparametric density estimators, Journal of Multivariate Analysis 14, 1-16.

Hall, P. and J.D. Hart, 1990, Bootstrap test for difference bewteen means in nonparametric regression, Journal of the American Statistical Association 85, 1039-1049.

Hall, P. and C.C. Heyde, 1980, Martingale Limit Theory and its Application (Academic Press: New-York). 
Hall, P., C. Huber and P.L. Speckman, 1997, Covariance-matched one-sided tests for the difference between functional means, Journal of the American Statistical Association 92(439), 1074-1083.

Härdle, W. and E. Mammen, 1993, Comparing nonparametric versus parametric regression fits, Annals of Statistics 21 (4), 1926-1947.

Härdle, W. and J.S. Marron, 1990, Semiparametric comparison of regression curves, Annals of Statistics 18 (1), 63-89.

Hart, J.D., 1997, Nonparametric Smoothing and Lack-of-Fit Tests (Springer Verlag: New-York).

Hoeffding, W., 1948, A class of statistics with asymptotically normal distribution, Annals of Mathematical Statistics 15, 293-325.

King, E.C., J.D. Hart and T.E. Wehrly, 1991, Testing the equality of two regression curves using linear smoothers, Statistics and Probability Letters 12, 239-247.

Koul, H.L. and A. Schick, 1997, Testing for the equality of two nonparametric regression curves, Journal of Statistical Planning and Inference 65, 293-314.

Kulasekera, K.B., 1995, Comparison of regression curves using quasi-residuals, Journal of the American Statistical Association 90(431), 1085-1093.

Lavergne, P. and Q.H. Vuong, 1996, Nonparametric selection of regressors: the nonnested case, Econometrica 64(1), 207-219.

Lavergne, P. and Q.H. Vuong, 2000, Nonparametric significance testing, Econometric Theory , to appear.

Li, Q., 1999, Consistent model specification tests for time series econometrics models, Journal of Econometrics 92(1), 101-147.

Mammen, E., 1992, When Does Bootstrap Work? Asymptotic Results and Simulations, Lecture Notes in Statistics, 77 (Springer-Verlag: New-York).

Munk, A. and H. Dette, 1998, Nonparametric comparison of several regression functions: exact and asymptotic theory, Annals of Statistics 26(6), 2339-2368.

Powell, J.L., H. Stock and T.M. Stoker, 1989, Semiparametric estimation of index coefficients, Econometrica 57, $1403-1430$.

Quade, D., 1982, Nonparametric analysis of covariance, Biometrics 38, 597-611.

Pinkse, C.A.P and P.M. Robinson, 1995, Pooling nonparametric estimates of regression funtions with a similar shape, in: G.S. Maddala, P.C.B. Phillips and T.N. Srinivasan, eds., Advances in Econometrics and Quantitative Economics: Essays in Honor of Professor C.R. Rao (B. Blackwell: Oxford) 172-197.

Robinson, P., 1988, Root-N consistent semiparametric regression, Econometrica 56, 931-954.

Scheffé, H., 1959, The Analysis of Variance (John Wiley \& Sons: New-York).

Yatchew, A., 1999, An elementary nonparametric differencing test of equality of regression functions, Economic Letters 62, 271-278. 
Young, S. G. and A. W. Bowman, 1995, Non-parametric analysis of covariance, Biometrics 51, 920-931.

Zheng, J.X., 1996, A consistent test of functional form via nonparametric estimation techniques, Journal of Econometrics 75 (2), 263-289. 
Table 1: Null and Linear Alternatives $-h \propto n^{-1 / 5}$

\begin{tabular}{|c|c|c|c|c|c|c|c|c|c|}
\hline \multirow{2}{*}{$\frac{n}{100}$} & \multirow{2}{*}{$\begin{array}{c}a \\
0.5\end{array}$} & \multicolumn{2}{|c|}{$D G P_{0}$} & \multicolumn{2}{|c|}{$D G P_{1}$} & \multicolumn{2}{|c|}{$D G P_{2}$} & \multicolumn{2}{|c|}{$D G P_{3}$} \\
\hline & & 0.001 & $(0.854)$ & 0.271 & $(0.935)$ & 0.966 & (1.116) & 2.725 & (1.334) \\
\hline & & $4.3 \%$ & $7.6 \%$ & $8.8 \%$ & $14.3 \%$ & $25.3 \%$ & $36.4 \%$ & $78.6 \%$ & $86.2 \%$ \\
\hline & & 1.546 & 1.111 & $9.8 \%$ & $17.9 \%$ & $28.1 \%$ & $42.2 \%$ & $80.6 \%$ & $88.2 \%$ \\
\hline & 1.0 & 0.061 & $(0.846)$ & 0.490 & (1.048) & 1.559 & (1.404) & 4.129 & (1.792) \\
\hline & & $4.4 \%$ & $8.3 \%$ & $13.1 \%$ & $19.7 \%$ & $43.1 \%$ & $53.6 \%$ & $91.3 \%$ & $94.4 \%$ \\
\hline & & 1.592 & 1.170 & $14.0 \%$ & $22.5 \%$ & $44.3 \%$ & $57.0 \%$ & $92.1 \%$ & $95.2 \%$ \\
\hline & 1.5 & 0.036 & $(0.876)$ & 0.582 & $(1.158)$ & 1.949 & (1.641) & 5.203 & $(2.159)$ \\
\hline & & $5.6 \%$ & $8.9 \%$ & $16.8 \%$ & $23.7 \%$ & $51.6 \%$ & $61.7 \%$ & $95.6 \%$ & $97.5 \%$ \\
\hline & & 1.696 & 1.176 & $15.9 \%$ & $26.0 \%$ & $50.3 \%$ & $64.0 \%$ & $95.3 \%$ & $97.8 \%$ \\
\hline & 2.0 & 0.034 & $(0.875)$ & 0.675 & $(1.230)$ & 2.280 & $(1.826)$ & 6.093 & $(2.469)$ \\
\hline & & $5.0 \%$ & $8.6 \%$ & $20.3 \%$ & $26.0 \%$ & $58.6 \%$ & $66.6 \%$ & $97.4 \%$ & $98.4 \%$ \\
\hline & & 1.642 & 1.171 & $20.3 \%$ & $28.8 \%$ & $58.6 \%$ & $68.8 \%$ & $97.4 \%$ & $98.7 \%$ \\
\hline & Chow test & $4.4 \%$ & $9.6 \%$ & $51.2 \%$ & $64.6 \%$ & $98.7 \%$ & $99.4 \%$ & $100.0 \%$ & $100.0 \%$ \\
\hline 200 & 0.5 & 0.031 & $(0.921)$ & 0.595 & (1.086) & 2.063 & (1.427) & 5.771 & (1.816) \\
\hline & & $5.1 \%$ & $9.5 \%$ & $16.3 \%$ & $23.2 \%$ & $59.8 \%$ & $69.7 \%$ & $98.7 \%$ & $99.5 \%$ \\
\hline & & 1.655 & 1.241 & $16.2 \%$ & $24.3 \%$ & $59.7 \%$ & $71.0 \%$ & $98.6 \%$ & $99.5 \%$ \\
\hline & 1.0 & 0.042 & $(0.899)$ & 0.932 & $(1.265)$ & 3.200 & $(1.870)$ & 8.667 & $(2.504)$ \\
\hline & & $5.0 \%$ & $8.8 \%$ & $24.5 \%$ & $34.1 \%$ & $79.8 \%$ & $85.4 \%$ & $99.9 \%$ & $100.0 \%$ \\
\hline & & 1.645 & 1.224 & $24.5 \%$ & $36.0 \%$ & $79.8 \%$ & $86.0 \%$ & $99.9 \%$ & $100.0 \%$ \\
\hline & 1.5 & 0.053 & $(0.910)$ & 1.175 & $(1.415)$ & 4.031 & $(2.213)$ & 10.838 & $(3.035)$ \\
\hline & & $5.9 \%$ & $9.7 \%$ & $32.5 \%$ & $41.3 \%$ & $86.4 \%$ & $90.3 \%$ & $100.0 \%$ & $100.0 \%$ \\
\hline & & 1.725 & 1.262 & $30.1 \%$ & $41.9 \%$ & $85.2 \%$ & $90.5 \%$ & $100.0 \%$ & $100.0 \%$ \\
\hline & 2.0 & 0.042 & $(0.900)$ & 1.354 & $(1.529)$ & 4.694 & $(2.493)$ & 12.624 & $(3.486)$ \\
\hline & & $5.6 \%$ & $9.3 \%$ & $36.0 \%$ & $44.9 \%$ & $89.8 \%$ & $92.2 \%$ & $100.0 \%$ & $100.0 \%$ \\
\hline & & 1.718 & 1.237 & $34.3 \%$ & $46.0 \%$ & $89.3 \%$ & $92.7 \%$ & $100.0 \%$ & $100.0 \%$ \\
\hline & Chow test & $5.3 \%$ & $9.8 \%$ & $92.8 \%$ & $96.6 \%$ & $100.0 \%$ & $100.0 \%$ & $100.0 \%$ & $100.0 \%$ \\
\hline
\end{tabular}

Each cell contains the empirical mean of the test statistic with its standard deviation in parentheses on the first line, empirical rejection percentages at $5 \%$ and $10 \%$ nominal levels on the second line, and size-corrected empirical rejection percentages at $5 \%$ and $10 \%$ levels on the third line. For the null hypothesis, i.e. $D G P_{0}$, empirical critical values for $5 \%$ and $10 \%$ levels are on the third line. 
Table 2: Sinus Alternatives $-h \propto n^{-1 / 5}$

\begin{tabular}{|c|c|c|c|c|c|c|c|c|c|}
\hline \multirow{2}{*}{$\frac{n}{100}$} & \multirow{2}{*}{$\frac{a}{0.5}$} & \multicolumn{2}{|c|}{$D G P_{4}$} & \multicolumn{2}{|c|}{$D G P_{5}$} & \multicolumn{2}{|c|}{$D G P_{6}$} & \multicolumn{2}{|c|}{$D G P_{7}$} \\
\hline & & 0.981 & $(1.069)$ & 0.877 & (1.136) & 0.956 & $(1.161)$ & 0.934 & $(1.142)$ \\
\hline & & $25.6 \%$ & $36.2 \%$ & $23.9 \%$ & $32.8 \%$ & $27.2 \%$ & $36.2 \%$ & $25.8 \%$ & $35.0 \%$ \\
\hline & & $28.3 \%$ & $42.1 \%$ & $26.4 \%$ & $38.2 \%$ & $29.2 \%$ & $40.6 \%$ & $28.2 \%$ & $40.7 \%$ \\
\hline & 1.0 & 1.241 & $(1.171)$ & 1.353 & $(1.375)$ & 1.529 & (1.467) & 1.510 & (1.444) \\
\hline & & $32.9 \%$ & $45.2 \%$ & $36.2 \%$ & $46.8 \%$ & $40.7 \%$ & $51.0 \%$ & $41.9 \%$ & $50.4 \%$ \\
\hline & & $34.2 \%$ & $48.8 \%$ & $37.6 \%$ & $49.7 \%$ & $42.0 \%$ & $54.2 \%$ & $42.9 \%$ & $53.9 \%$ \\
\hline & 1.5 & 0.943 & $(1.109)$ & 1.535 & $(1.549)$ & 1.853 & (1.678) & 1.861 & $(1.665)$ \\
\hline & & $23.1 \%$ & $32.5 \%$ & $42.0 \%$ & $50.3 \%$ & $48.3 \%$ & $57.5 \%$ & $48.5 \%$ & $57.7 \%$ \\
\hline & & $22.0 \%$ & $36.2 \%$ & $40.6 \%$ & $52.9 \%$ & $47.2 \%$ & $59.7 \%$ & $47.5 \%$ & $60.2 \%$ \\
\hline & 2.0 & 0.346 & (1.018) & 1.575 & (1.588) & 2.085 & $(1.827)$ & 2.142 & (1.835) \\
\hline & & $10.1 \%$ & $15.2 \%$ & $41.3 \%$ & $51.2 \%$ & $52.9 \%$ & $61.6 \%$ & $54.1 \%$ & $63.0 \%$ \\
\hline & & $10.1 \%$ & $17.1 \%$ & $41.4 \%$ & $54.3 \%$ & $53.0 \%$ & $64.5 \%$ & $54.3 \%$ & $65.7 \%$ \\
\hline & Chow test & $4.8 \%$ & $9.5 \%$ & $14.8 \%$ & $21.2 \%$ & $74.9 \%$ & $82.3 \%$ & $83.8 \%$ & $90.9 \%$ \\
\hline 200 & 0.5 & 2.037 & $(1.389)$ & 1.933 & $(1.427)$ & 2.078 & $(1.452)$ & 2.014 & $(1.429)$ \\
\hline & & $59.5 \%$ & $69.8 \%$ & $55.2 \%$ & 64. & $58.9 \%$ & 68 & $57.2 \%$ & $68.2 \%$ \\
\hline & & $59.3 \%$ & $70.7 \%$ & $55.0 \%$ & $65.8 \%$ & $58.7 \%$ & $69.5 \%$ & $56.9 \%$ & $68.9 \%$ \\
\hline & 1.0 & 2.626 & $(1.577)$ & 2.870 & $(1.825)$ & 3.182 & $(1.917)$ & 3.112 & $(1.882)$ \\
\hline & & $71.2 \%$ & $79.4 \%$ & $72.4 \%$ & $79.0 \%$ & $77.5 \%$ & $84.0 \%$ & $77.4 \%$ & $84.2 \%$ \\
\hline & & $71.1 \%$ & $80.4 \%$ & $72.4 \%$ & $80.3 \%$ & $77.5 \%$ & $84.9 \%$ & $77.4 \%$ & $84.9 \%$ \\
\hline & 1.5 & 2.347 & (1.498) & 3.395 & $(2.082)$ & 3.934 & $(2.242)$ & 3.892 & $(2.216)$ \\
\hline & & $64.7 \%$ & $74.4 \%$ & $79.0 \%$ & $84.3 \%$ & $85.3 \%$ & $89.6 \%$ & $84.2 \%$ & $88.8 \%$ \\
\hline & & $62.6 \%$ & $74.7 \%$ & $77.5 \%$ & $84.5 \%$ & $84.1 \%$ & $89.8 \%$ & $83.4 \%$ & $89.2 \%$ \\
\hline & 2.0 & 1.452 & $(1.299)$ & 3.588 & $(2.192)$ & 4.440 & $(2.491)$ & 4.470 & $(2.484)$ \\
\hline & & $38.8 \%$ & $48.4 \%$ & $79.5 \%$ & $85.8 \%$ & $87.3 \%$ & $91.4 \%$ & $87.9 \%$ & $91.5 \%$ \\
\hline & & $36.4 \%$ & $50.2 \%$ & $78.5 \%$ & $86.6 \%$ & $86.3 \%$ & $92.0 \%$ & $86.8 \%$ & $91.9 \%$ \\
\hline & Chow te & $5.5 \%$ & $10.1 \%$ & $13.4 \%$ & $21.0 \%$ & $93.3 \%$ & $95.9 \%$ & $99.6 \%$ & $100.0 \%$ \\
\hline
\end{tabular}

Each cell contains the empirical mean of the test statistic with its standard deviation in parentheses on the first line, empirical rejection percentages at $5 \%$ and $10 \%$ nominal levels on the second line, and size-corrected empirical rejection percentages at $5 \%$ and $10 \%$ levels on the third line. 
Table 3: Null and Linear Alternatives $-h \propto n^{-\lambda}, a=1$

\begin{tabular}{|c|c|c|c|c|c|c|c|c|c|}
\hline \multirow{2}{*}{$\frac{n}{100}$} & \multirow{2}{*}{$\frac{\lambda}{1 / 3}$} & \multicolumn{2}{|c|}{$D G P_{0}$} & \multicolumn{2}{|c|}{$D G P_{1}$} & \multicolumn{2}{|c|}{$D G P_{2}$} & \multicolumn{2}{|c|}{$D G P_{3}$} \\
\hline & & 0.013 & $(0.762)$ & 0.252 & $(0.852)$ & 0.874 & (1.037) & 2.398 & $(1.27$ \\
\hline & & 3 & & $6.7 \%$ & 11 & $21.1 \%$ & 32 & 72. & 80. \\
\hline & & 1.420 & 1.027 & $9.9 \%$ & $17.6 \%$ & $27.8 \%$ & $40.6 \%$ & $77.5 \%$ & $85.9 \%$ \\
\hline & $1 / 4$ & 0.019 & $(\cap 8 \cap$ & 0.365 & $(0.95$ & 1.248 & $(1.23$ & 3.394 & $(1.567)$ \\
\hline & & $3.8 \%$ & 7.5 & $10.7 \%$ & 16.0 & $33.8 \%$ & $44.9 \%$ & $86.0 \%$ & $90.5 \%$ \\
\hline & & 1.517 & 1.072 & $12.2 \%$ & $20.2 \%$ & $37.6 \%$ & $51.3 \%$ & $88.1 \%$ & $92.8 \%$ \\
\hline & $1 / 5$ & 0.061 & $(0.846)$ & 0.490 & $(1.0$ & 1.559 & (1.404) & 4.129 & $(1.792)$ \\
\hline & & $4.4 \%$ & . & $13.1 \%$ & $19.7 \%$ & $43.1 \%$ & $53.6 \%$ & $91.3 \%$ & $94.4 \%$ \\
\hline & & 1.592 & 1.170 & $14.0 \%$ & 22.5 & $44.3 \%$ & $57.0 \%$ & $92.1 \%$ & $95.2 \%$ \\
\hline & $1 / 6$ & 0.094 & $(0.880)$ & 0.578 & (1.112) & 1.777 & $(1.512)$ & 4.653 & (1.937) \\
\hline & & $5.7 \%$ & $9.2 \%$ & $16.2 \%$ & $22.9 \%$ & $48.4 \%$ & $59.0 \%$ & $94.1 \%$ & $96.2 \%$ \\
\hline & & 1.744 & 1.221 & $14.2 \%$ & 24.4 & $46.1 \%$ & 60. & $93.4 \%$ & $.5 \%$ \\
\hline 200 & $1 / 3$ & 0.029 & $(0.829)$ & 0.546 & (1.009) & 1.881 & (1.353) & 5.130 & $(1.741)$ \\
\hline & & $4.1 \%$ & $7.4 \%$ & $13.2 \%$ & $21.2 \%$ & $54.1 \%$ & $64.4 \%$ & $97.9 \%$ & $98.9 \%$ \\
\hline & & 1.486 & 1.096 & $16.8 \%$ & $26.5 \%$ & $58.8 \%$ & $70.7 \%$ & $98.5 \%$ & $99.3 \%$ \\
\hline & $1 / 4$ & 0.014 & $(0.899)$ & 0.745 & (1.189) & 2.630 & $(1.684)$ & 7.194 & $(2.197)$ \\
\hline & & $4.7 \%$ & $8.2 \%$ & $19.7 \%$ & $28.1 \%$ & $69.6 \%$ & $78.2 \%$ & $99.6 \%$ & $99.8 \%$ \\
\hline & & 1.622 & 1.138 & $20.2 \%$ & $31.8 \%$ & $70.1 \%$ & $80.7 \%$ & $99.6 \%$ & $99.9 \%$ \\
\hline & $1 / 5$ & 0.042 & $(0.899)$ & 0.932 & (1.265) & 3.200 & $(1.870)$ & 8.667 & $(2.504)$ \\
\hline & & $5.0 \%$ & $8.8 \%$ & $24.5 \%$ & $34.1 \%$ & $79.8 \%$ & $85.4 \%$ & $99.9 \%$ & $100.0 \%$ \\
\hline & & 1.645 & 1.224 & $24.5 \%$ & $36.0 \%$ & $79.8 \%$ & $86.0 \%$ & $99.9 \%$ & $100.0 \%$ \\
\hline & $1 / 6$ & 0.104 & $(0.913)$ & 1.112 & $(1.332)$ & 3.654 & $(2.022)$ & 9.772 & $(2.730)$ \\
\hline & & $6.0 \%$ & $9.6 \%$ & $30.5 \%$ & $40.4 \%$ & $84.2 \%$ & $89.1 \%$ & $99.9 \%$ & $100.0 \%$ \\
\hline & & 1.777 & 1.258 & $28.1 \%$ & $40.7 \%$ & $82.0 \%$ & $89.2 \%$ & $99.9 \%$ & $100.0 \%$ \\
\hline
\end{tabular}

Each cell contains the empirical mean of the test statistic with its standard deviation in parentheses on the first line, empirical rejection percentages at $5 \%$ and $10 \%$ nominal levels on the second line, and size-corrected empirical rejection percentages at $5 \%$ and $10 \%$ levels on the third line. For the null hypothesis, i.e. $D G P_{0}$, empirical critical values for $5 \%$ and $10 \%$ levels are on the third line. 
Table 4: Sinus Alternatives $-h \propto n^{-\lambda}, a=1$

\begin{tabular}{|c|c|c|c|c|c|c|c|c|c|}
\hline$n$ & $\lambda$ & \multicolumn{2}{|c|}{$D G P_{4}$} & \multicolumn{2}{|c|}{$D G P_{5}$} & \multicolumn{2}{|c|}{$D G P_{6}$} & \multicolumn{2}{|c|}{$D G P_{7}$} \\
\hline \multirow[t]{3}{*}{100} & \multirow[t]{3}{*}{$1 / 3$} & 0.822 & $(1.006)$ & 0.826 & $(1.080)$ & 0.886 & (1.098) & 0.860 & $(1.075)$ \\
\hline & & $19.6 \%$ & 29 & $21.5 \%$ & 30. & 2 & 33 & $22.2 \%$ & 3 \\
\hline & & $25.3 \%$ & $37.7 \%$ & $26.6 \%$ & $38.1 \%$ & $29.1 \%$ & $40.2 \%$ & $28.3 \%$ & $39.0 \%$ \\
\hline & \multirow[t]{3}{*}{$1 / 4$} & 1.079 & (1.122) & 1.127 & (1.261) & 1.245 & (1.313) & 1.217 & $(1.283)$ \\
\hline & & $27.9 \%$ & $38.1 \%$ & $29.8 \%$ & $40.2 \%$ & $33.3 \%$ & 43. & $33.0 \%$ & $42.7 \%$ \\
\hline & & $31.4 \%$ & $45.5 \%$ & $32.9 \%$ & $46.6 \%$ & $37.3 \%$ & $48.9 \%$ & $36.9 \%$ & $48.4 \%$ \\
\hline & \multirow[t]{3}{*}{$1 / 5$} & 1.241 & (1.171) & 1.353 & $(1.375)$ & 1.529 & (1.467) & 1.510 & $(1.444)$ \\
\hline & & $32.9 \%$ & $45.2 \%$ & $36.2 \%$ & $46.8 \%$ & $40.7 \%$ & $51.0 \%$ & $41.9 \%$ & $50.4 \%$ \\
\hline & & $34.2 \%$ & $48.9 \%$ & $37.6 \%$ & $49.8 \%$ & $42.0 \%$ & $54.2 \%$ & $42.9 \%$ & $54.0 \%$ \\
\hline & \multirow[t]{3}{*}{$1 / 6$} & 1.327 & $(1.179)$ & 1.492 & (1.464) & 1.717 & $(1.576)$ & 1.711 & $(1.553)$ \\
\hline & & $35.8 \%$ & $46.6 \%$ & $41.7 \%$ & 50. & $45.8 \%$ & 56 & $45.8 \%$ & $56.4 \%$ \\
\hline & & $32.7 \%$ & $48.9 \%$ & $39.0 \%$ & 52.0 & $42.8 \%$ & 58. & $43.6 \%$ & $57.9 \%$ \\
\hline \multirow[t]{3}{*}{200} & \multirow[t]{3}{*}{$1 / 3$} & 1.759 & $(1.324)$ & 1.800 & $(1.355)$ & 1.925 & (1.378) & 1.855 & $(1.358)$ \\
\hline & & $49.6 \%$ & $60.5 \%$ & $51.2 \%$ & $61.8 \%$ & $54.7 \%$ & $65.1 \%$ & $52.0 \%$ & $63.8 \%$ \\
\hline & & $53.9 \%$ & $65.6 \%$ & $55.8 \%$ & $67.1 \%$ & $59.7 \%$ & $70.1 \%$ & $57.1 \%$ & $69.3 \%$ \\
\hline & \multirow[t]{3}{*}{$1 / 4$} & 2.314 & $(1.535)$ & 2.449 & (1.677) & 2.658 & $(1.722)$ & 2.577 & $(1.690)$ \\
\hline & & $63.8 \%$ & $72.7 \%$ & $64.1 \%$ & $73.8 \%$ & $70.3 \%$ & $78.4 \%$ & $68.2 \%$ & $77.2 \%$ \\
\hline & & $64.2 \%$ & $76.0 \%$ & $64.7 \%$ & $77.3 \%$ & $70.7 \%$ & $80.5 \%$ & $68.9 \%$ & $80.5 \%$ \\
\hline & \multirow[t]{3}{*}{$1 / 5$} & 2.626 & (1.577) & 2.870 & (1. & 3.182 & (1. & 3.112 & $(1$. \\
\hline & & $71.2 \%$ & $79.4 \%$ & $72.4 \%$ & $79.0 \%$ & $77.5 \%$ & $84.0 \%$ & $77.4 \%$ & $84.2 \%$ \\
\hline & & $71.2 \%$ & $80.4 \%$ & $72.4 \%$ & $80.3 \%$ & $77.5 \%$ & $84.9 \%$ & $77.4 \%$ & $84.9 \%$ \\
\hline & \multirow[t]{3}{*}{$1 / 6$} & 35 & $(1.5$ & 62 & $(1.9$ & .584 & $(2.0$ & 3.535 & $(2.026)$ \\
\hline & & $75.3 \%$ & $83.5 \%$ & $76.7 \%$ & $83.7 \%$ & $82.4 \%$ & $87.5 \%$ & $81.8 \%$ & $86.6 \%$ \\
\hline & & $72.5 \%$ & $84.0 \%$ & $73.9 \%$ & $84.0 \%$ & $79.4 \%$ & $88.0 \%$ & $79.9 \%$ & $87.0 \%$ \\
\hline
\end{tabular}

Each cell contains the empirical mean of the test statistic with its standard deviation in parentheses on the first line, empirical rejection percentages at $5 \%$ and $10 \%$ nominal levels on the second line, and size-corrected empirical rejection percentages at $5 \%$ and $10 \%$ levels on the third line. 